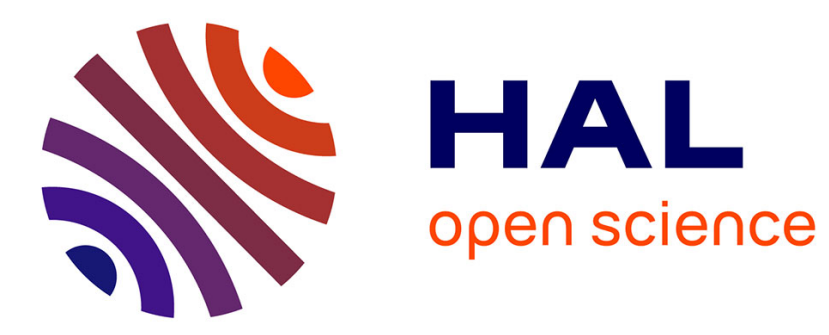

\title{
A periodically forced piecewise linear oscillator
}

Steven W Shaw, Philip J Holmes

\section{To cite this version:}

Steven W Shaw, Philip J Holmes. A periodically forced piecewise linear oscillator. Journal of Sound and Vibration, 1983, 10.1016/0022-460X(83)90407-8 . hal-01509017

\section{HAL Id: hal-01509017 https://hal.science/hal-01509017}

Submitted on 15 Apr 2017

HAL is a multi-disciplinary open access archive for the deposit and dissemination of scientific research documents, whether they are published or not. The documents may come from teaching and research institutions in France or abroad, or from public or private research centers.
L'archive ouverte pluridisciplinaire HAL, est destinée au dépôt et à la diffusion de documents scientifiques de niveau recherche, publiés ou non, émanant des établissements d'enseignement et de recherche français ou étrangers, des laboratoires publics ou privés. 


\title{
A PERIODICALLY FORCED PIECEWISE LINEAR OSCILLATOR $\dagger$
}

\author{
S. W. Shaw And P. J. Holmes \\ Department of Theoretical and Applied Mechanics, Cornell University, Ithaca, \\ New York 14853, U.S.A.
}

\begin{abstract}
A single-degree of freedom non-linear oscillator is considered. The non-linearity is in the restoring force and is piecewise linear with a single change in slope. Such oscillators provide models for mechanical systems in which components make intermittent contact. A limiting case in which one slope approaches infinity, an impact oscillator, is also considered. Harmonic, subharmonic, and chaotic motions are found to exist and the bifurcations leading to them are analyzed.
\end{abstract}

\section{INTRODUCTION}

Mechanical systems in which moving components make intermittent contact with each other often give rise to equations of motion containing piecewise linear terms. Such systems have been the subject of several investigations, because of the apparently simple nature of the non-linearity. Standard texts on non-linear oscillations such as those of Minorsky [1] and Andronov et al. [2] contain analyses of these systems, although only free oscillations are considered. In contrast, periodically forced systems with non-linear restoring forces are studied in the present paper. Single degree of freedom oscillators of this type were studied by Maezawa and Furukawa [3] and Dragoni and Repaci [4], who considered symmetric restoring forces. The non-symmetric case with linear damping is to be considered in the present paper. Maezawa [5] and Maezawa et al. [6] previously studied the harmonic and superharmonic response of such a system using a Fourier series method. Klotter [7] obtained harmonic motions of several piecewise linear systems using a Galerkin method, but the stability of these harmonic solutions was not discussed. Thompson [8] has studied harmonic and subharmonic motions using numerical methods. An experimental investigation of both symmetric and non-symmetric restoring forces has been done by Robinson [9], who has observed many of the phenomena we analyze in this paper. Moreover, such piecewise linear systems have not been used to model the motion of beams with non-linear boundary conditions [10-12].

When one stiffness approaches infinity the system becomes an impact oscillator. Senator [13] studied such a system with a constant restoring force and energy loss upon impact. He discussed single impact periodic motions and their stability. Holmes [14] considered a similar system (a mass bouncing on a vibrating table) and found not only harmonic and subharmonic motions, but also bounded non-periodic "chaotic" ones. The impact oscillator considered here was studied in detail using numerical methods by Thompson $[15,16]$. He found cascades of period doubling bifurcations from harmonic and subharmonic solutions, apparently leading to non-periodic motions. These period doubling bifurcations seem similar to those found in one dimensional maps $[17,18]$.

† This work was supported by NSF Grant MEA-80-17570. 


\section{DESCRIPTION OF THE SYSTEM}

\subsection{THE GENERAL SYSTEM}

Consider the simple system shown in Figure 1. A mass $m$ is attached to a linear spring of stiffness $k_{1}$ and a linear dashpot with damping factor $c$. When the displacement, $x$,

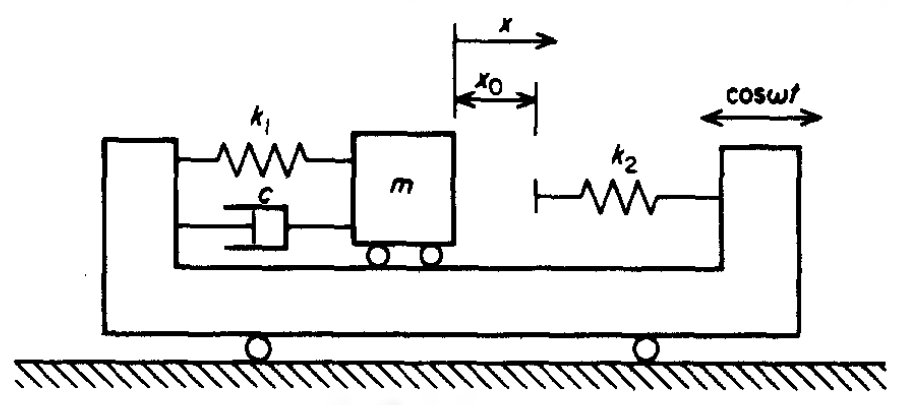

Figure 1. The physical system.

exceeds a certain value, $x_{0}$, a second linear spring, $k_{2}$, contacts $m$. Without loss of generality one can assume $x_{0} \geqslant 0$. The two springs give rise to an overall restoring force which is piecewise linear. When the system is externally excited by a harmonic force, the non-dimensionalized equation of motion may be written as

$$
\ddot{x}+2 \alpha \dot{x}+H(x)=\beta \cos (\omega t),
$$

where

$$
H(x)=\left\{\begin{array}{ll}
x, & x<x_{0} \\
\tilde{\omega}^{2} x+\left(1-\tilde{\omega}^{2}\right) x_{0}, & x \geqslant x_{0}
\end{array}\right\},
$$

$\tilde{\omega}^{2}$ is the stiffiness ratio $\left(k_{1}+k_{2}\right) / k_{1}=\omega_{+}^{2} / \omega_{-}^{2}, \alpha$ is the damping coefficient, which we shall generally assume to be less than 1 (subcritical), $\beta$ is the forcing amplitude, and $\omega$ is the forcing frequency. An overdot indicates differentiation with respect to the nondimensional time $t$. Figure 2(a) shows the function $H(x)$.
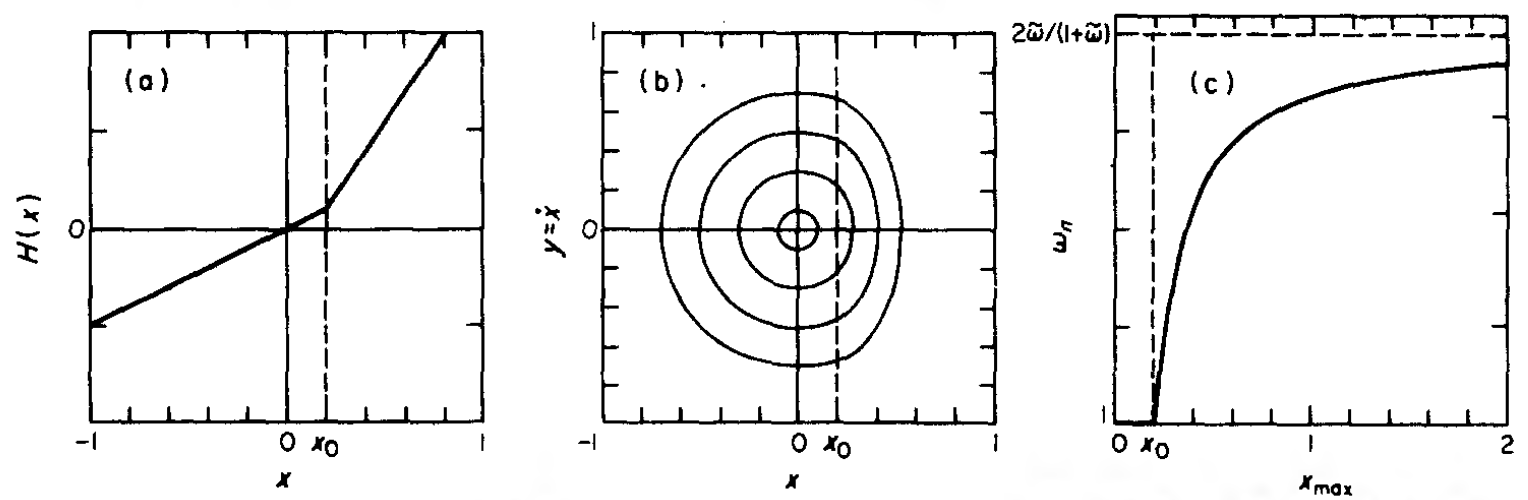

Figure 2. (a) Restoring force $H(x)$ us, displacement $x$; (b) undamped ( $\alpha=0)$, unforced $(\beta=0)$ phase portrait, velocity $(y)$ vs. displacement; (c) frequency $\omega_{n} v s$. amplitude $x_{\text {MAX }}$.

Local solutions of this equation are known explicitly on each side of $x=x_{0}$. Such solutions can be repeatedly matched at $x=x_{0}$ to obtain a global solution of equation (1). Piecing together these known solutions is not directly possible however, since, as will be seen, the times of flight in each region cannot be found in closed form. 
The two equations are

$$
\begin{gathered}
\ddot{x}+2 \alpha \dot{x}+x=\beta \cos (\omega t) \text { for } x \leqslant x_{0}, \\
\ddot{x}+2 \alpha \dot{x}+\tilde{\omega}^{2} x+\left(1-\tilde{\omega}^{2}\right) x_{0}=\beta \cos (\omega t) \text { for } x \geqslant x_{0} .
\end{gathered}
$$

The solution to equation (3) based at $x\left(t_{0}\right)=x_{0}$ and $\dot{x}\left(t_{0}\right)=y_{0}$, for $\alpha<1$, is

$$
\begin{aligned}
x_{+}\left(t ; t_{0}, y_{0}\right)= & \mathrm{e}^{-\alpha\left(t-t_{0}\right)}\left[A_{+} \cos \left(\Omega_{+}\left(t-t_{0}\right)\right)+B_{+} \sin \left(\Omega_{+}\left(t-t_{0}\right)\right]\right. \\
& +\gamma_{+} \cos (\omega t)+\delta_{+} \sin (\omega t)-x_{0}\left(1-\tilde{\omega}^{2}\right) / \tilde{\omega}^{2},
\end{aligned}
$$

where $\gamma_{+}=\left(\tilde{\omega}^{2}-\omega^{2}\right) \beta / \Delta_{+}, \delta_{+}=(2 \alpha \omega) \beta / \Delta_{+}, \Delta_{+}=\left(\tilde{\omega}^{2}-\omega^{2}\right)^{2}+(2 \alpha \omega)^{2}, \Omega_{+}^{2}=\tilde{\omega}^{2}-\alpha^{2}$, $A_{+}=-\gamma_{+} c_{0}-\delta_{+} s_{0}+x_{0} / \tilde{\omega}^{2}, \quad B_{+}=\left(1 / \Omega_{+}\right)\left[y_{0}+\alpha x_{0} / \tilde{\omega}^{2}+s_{0}\left(\gamma_{+} \omega-\delta_{+} \alpha\right)-c_{0}\left(\gamma_{+} \alpha+\delta_{+} \omega\right)\right]$, $\mathrm{c}_{0}=\cos \left(\omega t_{0}\right)$, and $\mathrm{s}_{0}=\sin \left(\omega t_{0}\right)$.

Similarly the solution of equation (2) based at $x\left(t_{1}\right)=x_{0}$ and $\dot{x}\left(t_{1}\right)=y_{1}$ is

$$
x_{-}\left(t ; t_{1}, y_{1}\right)=\mathrm{e}^{-\alpha\left(t-t_{1}\right)}\left[A_{-} \cos \left(\Omega_{-}\left(t-t_{1}\right)\right)+B_{-} \sin \left(\Omega_{-}\left(t-t_{1}\right)\right)\right]+\gamma_{-} \cos (\omega t)+\delta_{-} \sin (\omega t),
$$

where $\gamma_{-}=\left(1-\omega^{2}\right) \beta / \Delta_{-}, \delta_{-}=(2 \alpha \omega) \beta / \Delta_{-}, \Delta_{-}=\left(1-\omega^{2}\right)^{2}+(2 \alpha \omega)^{2}, \Omega_{-}^{2}=1-\alpha^{2}, A_{--}=$ $x_{0}-\gamma_{-} c_{1}-\delta_{-} s_{1}, \quad B_{-}=\left(1 / \Omega_{-}\right)\left[y_{1}+\alpha x_{0}+s_{1}\left(\gamma_{-} \omega-\delta_{-} \alpha\right)-c_{1}\left(\gamma_{-} \alpha+\delta_{-} \omega\right)\right], \quad c_{1}=\cos \left(\omega t_{1}\right)$, and $s_{1}=\sin \left(\omega t_{1}\right)$.

The difficulty in joining solutions (4) and (5) together to obtain the global solution is that the crossing times (when $x\left(t_{i}\right)=x_{0}$ ) are not known explicitly. These times are roots of the equations

$$
x_{+}\left(t ; t_{0}, y_{0}\right)=x_{0} \text { and } x_{-}\left(t ; t_{1}, y_{1}\right)=x_{0} .
$$

When $\beta=0$ and $\alpha=0$, the unforced, undamped system has a phase portrait like that shown in Figure 2(b). The phase plane is filled with a continuous family of closed orbits. For $x_{\max }<x_{0}$ the orbits are simple harmonic motions of period $2 \pi$. For $x_{\max }>x_{0}$ the orbits consist of two pieces of ellipses jointed at $x=x_{0}$. The natural frequency of oscillation as a function of amplitude is shown in Figure 2(c). It is 1 up to $x_{\max }=x_{0}$ and then begins to increase. As the amplitude becomes large, the frequency approaches an asymptotic value, since the gap (between $x=0$ and $x_{0}$ ) becomes negligible. This asymptotic value is obtained from the case $x_{0}=0$, considered below, since for large amplitude motions $\left|x_{\max }\right| \gg\left|x_{0}\right|$.

The reader should note that orbits are once differentiable along $x=x_{0}$. This result is obtained directly from equation (1), and follows from the Lipschitz-continuity of the function $H(x)$. Henceforth we shall adopt the notation that $y=\dot{x}$.

\subsection{THE $x_{0}=0$ CASE}

In the $x_{0}=0$ case the forcing amplitude may be scaled out by letting $x \rightarrow \beta x$. Also in this case the unforced system has a natural frequency which is independent of the amplitude of oscillation. This is an important difference between this and most other non-linear systems (including the $x_{0} \neq 0$ case). The damped natural frequency in this case becomes

$$
\Omega_{n}=2 \Omega_{+} \Omega_{-} /\left(\Omega_{+}+\Omega_{-}\right),
$$

where $\Omega_{-}^{2}=1-\alpha^{2}$ and $\Omega_{+}^{2}=\tilde{\omega}^{2}-\alpha^{2}$. The undamped natural frequency is obtained by setting $\alpha=0$ in equation (8) and is

$$
\omega_{n}=2 \tilde{\omega} /(1+\tilde{\omega}) \text {. }
$$

The undamped, unforced phase plane for this case is shown in Figure 3. 


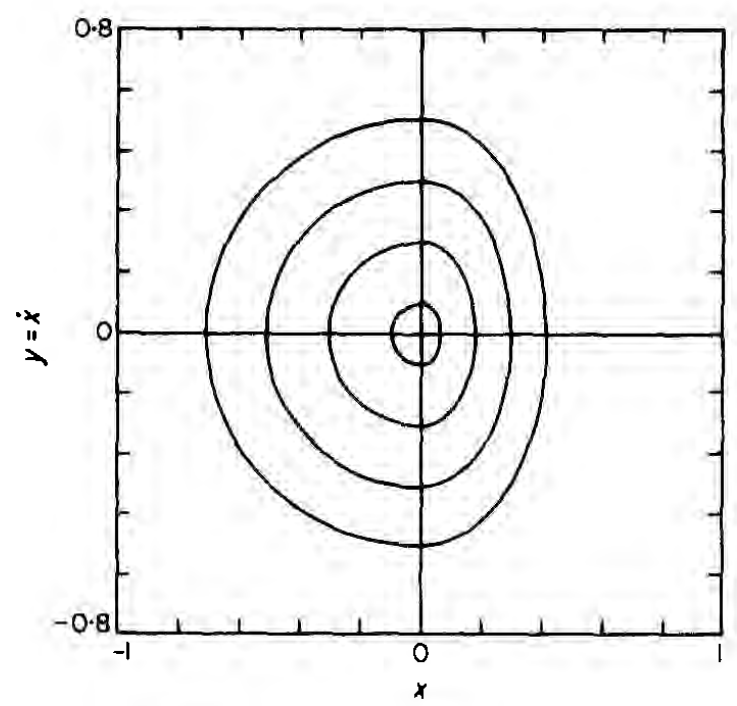

Figure 3. Phase portrait for $\alpha=0, \beta=0, x_{0}=0$.

\subsection{THE IMPACT LIMIT}

When $k_{2} \rightarrow \infty$ the resulting system will be referred to as an impact oscillator $[15,16]$. Letting $\varepsilon=1 / \tilde{\omega}$ and rescaling equation (1) yields, for $x>x_{0}$,

$$
\ddot{x}+2 \alpha \varepsilon \dot{x}+x=\varepsilon^{2} \beta \cos (\bar{\omega} \tau)+x_{0}\left(1-\varepsilon^{2}\right),
$$

where $\bar{\omega}=\omega / \omega_{+}$and $\tau=\omega_{+}$. The solution in this rescaled time variable is (cf. equation (4))

$$
x(\tau)=x_{0}+y_{0}\left(1-\alpha \varepsilon\left(\tau-\tau_{0}\right)\right) \sin \left(\tau-\tau_{0}\right)+O\left(\varepsilon^{2}\right) .
$$

The total time spent in $x>x_{0}$ may be computed by solving for the first root $\tau_{1}>\tau_{0}$ of $x\left(\tau_{1}\right)=x_{0}$. Letting $\tau_{1}=T_{10}+\varepsilon T_{11}+O\left(\varepsilon^{2}\right)$ and solving, one obtains $\tau_{1}=\tau_{0}+\pi+O\left(\varepsilon^{2}\right)$. Hence, from equation (11), if the velocity at impact is $y_{0}$, immediately after the impact it is

$$
y_{1}=\dot{x}\left(\tau_{1}\right)=-(1-\alpha \varepsilon \pi) y_{0}+O\left(\varepsilon^{2}\right) .
$$

The factor $(1-\alpha \varepsilon \pi)$ is the leading part of the Taylor series expansion of the exponential damping decay $\mathrm{e}^{-\alpha \varepsilon \pi}$ and represents a loss of energy during impact. In what follows we shall denote this coefficient of restitution as $r$ and write

$$
y_{1}=-r y_{0} \text {, }
$$

thus obtaining the standard impact rule (cf. reference [19]). Although as derived here $r=1-O(\varepsilon)$, we shall generalize and allow $r$ to range from 0 to 1 . This allows us to account for other losses during the impact.

\section{THE POINCARÉ SECTION AND RETURN MAP}

To study equations of this type we shall employ the method of a Poincare section. For a one degree of freedom periodically forced oscillator one has a three dimensional extended phase space with co-ordinates $(x, y, t)$. The vector field defined by equation (1) is easily seen to be $2 \pi / \omega$ periodic in $t$. A natural place to slice this space is at the points of discontinuity in stiffness: i.e., at $x=x_{0}$. We define the Poincaré section as

$$
\Sigma=\left\{(x, y, t) \mid x=x_{0}, y>0\right\} \text {. }
$$


Orbits in the phase space will be studied by considering the mapping

$$
P: \Sigma \rightarrow \Sigma
$$

induced by solutions of equation (1). Figure 4 shows the phase space and the section $\Sigma$. This section is used since the solutions of the linear equations are known explicitly on

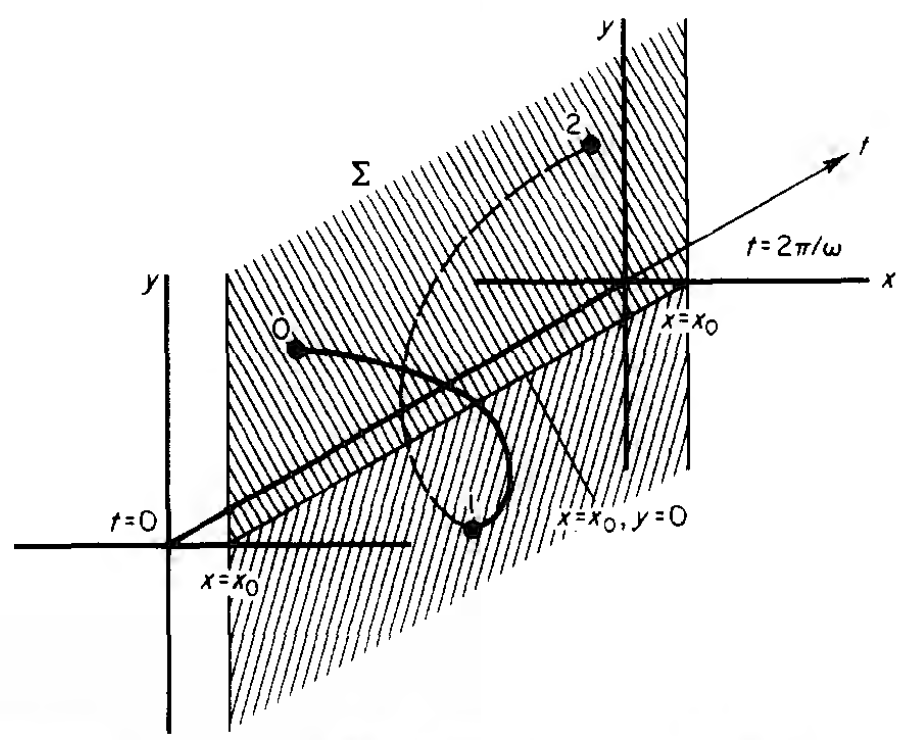

Figure 4. Phase space $(x, y, t)$ showing the Poincaré section $\Sigma$. Note the $2 \pi / \omega$ periodicity in $t$.

each side of $\Sigma$. It should be noted that the flow of the differential equation is everywhere transverse to $\Sigma$. This is easily seen by considering the vector field in $(x, y, t)$ space on $\Sigma$ given by

$$
\dot{x}=y, \quad \dot{y}=-2 \alpha y+x_{0}+\beta \cos (\omega t), \quad \dot{t}=1 .
$$

for $y>0, \dot{x} \neq 0$ and thus the flow is transverse to $\Sigma$. However, $P$ is not always well defined and is not generally "onto", since some points in $\Sigma$ are mapped onto the line $x=y=0$. This leads to discontinuities in the mapping, as will be seen. [20]

The section $\Sigma$ considered here should be distinguished from the more common section

$$
\Sigma^{t_{0}}=\left\{(x, y, t) \mid t=t_{0}, \bmod 2 \pi / \omega\right\} .
$$

On $\Sigma^{t_{0}}$ a point which is mapped on to itself after $k$ iterates of the return map $P^{t_{0}}: \Sigma^{t_{0}} \rightarrow \Sigma^{t_{0}}$ is known to correspond to a (subharmonic) orbit with period $k(2 \pi / \omega)$ for the differential equation. In contrast, points on $\Sigma$ are never mapped back to themselves, since the $t$ (time) co-ordinate continues to increase linearly. Periodic (subharmonic) orbits correspond to points $(\bar{y}, \bar{t})$ with images $\mathbf{P}(\bar{y}, \bar{t})=(\bar{y}, \bar{t}+2 \pi k / \omega)$; if $k=1$ one has a harmonic (period $2 \pi / \omega)$ response. Note that orbits of the same period $k(2 \pi / \omega)$ might contain different numbers of impacts in the same time $2 \pi k / \omega$. In what follows we are mainly concerned with single impact, period $k$ motions. However, in both cases, the stability of periodic points of the return map is the same as the stability of the corresponding orbits, and bifurcations of these orbits may be studied by considering bifurcations of the periodic points of the return map.

The mapping $\mathbf{P}$ cannot be written down explicitly. Consider an orbit starting at point $0=\left(t_{0}, y_{0}\right)$ as shown in Figure 4. From point 0 to point $1=\left(t_{1}, y_{1}\right)$ the motion is governed by equation (4) (note that point 1 is not in $\Sigma$ but is in $\left.\left\{(x, y, t) \mid x=x_{0}, y<0\right\}\right)$. Here one 
must solve equation (6) for the $x_{0}$ crossing time $t_{1}$,

$$
x_{+}\left(t_{1} ; t_{0}, y_{0}\right)=x_{0},
$$

where $t_{1}$ is the first root larger than $t_{0}$. Once $t_{1}$ has been determined then one can immediately compute

$$
y_{1}=\dot{x}_{+}\left(t_{1} ; t_{0}, y_{0}\right)
$$

and point 1 has been determined. A similar procedure but with the solution $x_{-}\left(t ; t_{1}, y_{1}\right)$ of equation (5) takes the orbit from point 1 to point 2 . The mapping $\mathbf{P}$ can then be written schematically as a difference equation in the form

$$
t_{n+2}=f\left(t_{n}, y_{n}\right), \quad y_{n+2}=g\left(t_{n}, y_{n}\right)
$$

Here the functions $f$ and $g$ are necessarily $2 \pi / \omega$ periodic in $t_{n}$, but since their nature depends on the roots of transcendental equations, they cannot be written down explicitly. This implies that, in general, periodic points of the map cannot be analytically determined. It will be seen, however, that in the impact limit certain periodic orbits can be found analytically, and even in the general case one can study the behavior of some of the periodic points without explicitly finding them. This is done by examining their stability and the bifurcations they undergo.

\section{PERIODIC ORBITS AND LOCAL BIFURCATIONS}

\subsection{GENERAL CASE}

The stability of a periodic point is determined by the eigenvalues of the first derivative of the map evaluated at that point. Bifurcations occur when the linearized map is degenerate: i.e., at least one eigenvalue has unit modulus [21]. We denote a period one point by $(\bar{t}, \bar{y})$ where

$$
\bar{t}=f(\bar{t}, \bar{y})-2 \pi / \omega, \quad \bar{y}=g(\bar{t}, \bar{y}) .
$$

The first derivative of the map DP is given by

$$
\mathbf{D P}=\left[\begin{array}{ll}
\frac{\partial f}{\partial t} & \frac{\partial f}{\partial y} \\
\frac{\partial g}{\partial t} & \frac{\partial g}{\partial y}
\end{array}\right] \stackrel{\text { def }}{=}\left[\frac{\partial(f, g)}{\partial(t, y)}\right] .
$$

DP can be computed directly by using implicit differentiation. We outline that calculation here.

Differentiating equation (18) with respect to $t_{0}$ and $y_{0}$, using equation (4), gives $\partial t_{1} / \partial\left(t_{0}, y_{0}\right)$, as follows:

$$
\frac{\partial t_{1}}{\partial t_{0}}=\frac{\mathrm{e}^{-\alpha\left(t_{1}-t_{0}\right)}}{\Omega_{+} y_{1}}\left[\Omega_{+} y_{0} c_{+}+N_{0} \mathrm{~s}_{+}\right], \quad \frac{\partial t_{1}}{\partial y_{0}}=\frac{-\mathrm{e}^{-\alpha\left(t_{1}-t_{0}\right)}}{\Omega_{+} y_{1}} \mathrm{~s}_{+},
$$

where

$$
c_{+}=\cos \left(\Omega_{+}\left(t_{1}-t_{0}\right)\right), \quad s_{+}=\sin \left(\Omega_{+}\left(t_{1}-t_{0}\right)\right), \quad \text { and } \quad N_{0}=\beta c_{0}-\alpha y_{0}-x_{0} .
$$

Next, taking derivatives of equation (19) using the first time derivative of equation (4) and equations (16), (23) and (24) gives

$$
\frac{\partial y_{1}}{\partial t_{0}}=\frac{\mathrm{e}^{-\alpha\left(t_{1}-t_{0}\right)}}{\Omega_{+} y_{1}}\left[\left(N_{1} N_{0}+\Omega_{+}^{2} y_{0} y_{1}\right) \mathrm{s}_{+}+c_{+} \Omega_{+}\left(y_{0} N_{1}-y_{1} N_{0}\right)\right]
$$




$$
\frac{\partial y_{1}}{\partial y_{0}}=\frac{\mathrm{e}^{-\alpha\left(t_{1}-t_{0}\right)}}{\Omega_{+} y_{1}}\left[\Omega_{+} y_{1} c_{+}-N_{1} \mathbf{s}_{+}\right],
$$

where $N_{1}=\beta c_{1}-\alpha y_{1}-x_{0}$, and $c_{1}=\cos \left(\omega t_{1}\right)$. Equations (23)-(26) give the four components of $\left[\partial\left(t_{1}, y_{1}\right) / \partial\left(t_{0}, y_{0}\right)\right]$.

A similar calculation carried out between points 1 and 2 yields $\left[\partial\left(t_{2}, y_{2}\right) / \partial\left(t_{1}, y_{1}\right)\right]$. One simply replaces the subscripts 0,1 , and + by 1,2 , and - , respectively, in equations (23)-(26).

From the chain rule one has

$$
\left[\frac{\partial(f, g)}{\partial\left(t_{0}, y_{0}\right)}\right]=\left[\frac{\partial\left(t_{2}, y_{2}\right)}{\partial\left(t_{0}, y_{0}\right)}\right]=\left[\frac{\partial\left(t_{2}, y_{2}\right)}{\partial\left(t_{1}, y_{1}\right)}\right]\left[\frac{\partial\left(t_{1}, y_{1}\right)}{\partial\left(t_{0}, y_{0}\right)}\right],
$$

and equation (27) then gives the desired components of DP:

$$
\begin{aligned}
& \partial f / \partial t_{0}=\partial t_{2} / \partial t_{0} \\
& =\left[\mathrm{e}^{-\alpha\left(t_{2}-t_{0}\right)} / \Omega_{+} \Omega_{-} y_{2}\right]\left[-\mathrm{s}_{+} \mathrm{s}_{-}\left(\Omega_{+}^{2} y_{0}\right)+\mathrm{s}_{-} \mathrm{c}_{+}\left(\Omega_{+} N_{0}\right)+\mathrm{s}_{+} \mathrm{c}_{-}\left(\Omega_{-} N_{0}\right)+\mathrm{c}_{+} \mathrm{c}_{-}\left(\Omega_{+} \Omega_{-} y_{0}\right)\right], \\
& \partial f / \partial y_{0}=\partial t_{2} / \partial y_{0}=\left[\mathrm{e}^{-\alpha\left(t_{2}-t_{0}\right)} / \Omega_{+} \Omega_{-} y_{2}\right]\left[-s_{-} c_{+} \Omega_{+}-s_{+} c_{-} \Omega_{-}\right], \\
& \partial g / \partial t_{0}=\partial y_{2} / \partial t_{0}=\left[\mathrm{e}^{-\alpha\left(t_{2}-t_{0}\right)} / \Omega_{+} \Omega_{-} y_{2}\right]\left[\mathrm{s}_{+} \mathrm{s}_{-}\left(\Omega_{-}^{2} y_{2} N_{0}-\Omega_{+}^{2} y_{0} N_{2}\right)\right. \\
& +\Omega_{+} \mathrm{s}_{-} \mathrm{c}_{+}\left(N_{0} N_{2}+\Omega_{-}^{2} y_{0} y_{2}\right)+\Omega_{-} \mathrm{s}_{+} \mathrm{c}_{-}\left(N_{0} N_{2}+\Omega_{+}^{2} y_{0} y_{2}\right) \\
& \left.+\Omega_{+} \Omega_{-} c_{+} c_{-}\left(y_{0} N_{2}-y_{2} N_{0}\right)\right] \text {, } \\
& \partial g / \partial y_{0}=\partial y_{2} / \partial y_{0}=\left[\mathrm{e}^{-\alpha\left(t_{2}-t_{0}\right)} / \Omega_{+} \Omega_{-} y_{2}\right]\left[-s_{+} s_{-}\left(\Omega_{-}^{2} y_{2}\right)-s_{-} c_{+}\left(\Omega_{+} N_{2}\right)-s_{+} c_{-}\left(\Omega_{-} N_{2}\right)\right. \\
& \left.+c_{+} c_{-}\left(\Omega_{+} \Omega_{-} y_{2}\right)\right] \text {; }
\end{aligned}
$$

where $c_{-}=\cos \left(\Omega_{-}\left(t_{2}-t_{1}\right)\right)$, and $s_{-}=\sin \left(\Omega_{-}\left(t_{2}-t_{1}\right)\right)$. This matrix has the determinant

$$
D=\left(y_{0} / y_{2}\right) \mathrm{e}^{-2 \alpha\left(t_{2}-t_{0}\right)},
$$

and trace

$$
\begin{aligned}
T= & {\left[\mathrm{e}^{-\alpha\left(t_{2}-t_{0}\right)} / \Omega_{+} \Omega_{-} y_{2}\right]\left[-\mathrm{s}_{+} \mathrm{s}_{-}\left(y_{0} \Omega_{+}^{2}+y_{2} \Omega_{-}^{2}\right)+\mathrm{s}_{-} \mathrm{c}_{+} \Omega_{+}\left(N_{0}-N_{2}\right)\right.} \\
& \left.+\mathrm{s}_{+} \mathrm{c}_{-} \Omega_{-}\left(N_{0}-N_{2}\right)+\mathrm{c}_{+} \mathrm{c}-\Omega_{+} \Omega_{-}\left(y_{0}+y_{2}\right)\right] .
\end{aligned}
$$

One can now evaluate $D$ and $T$ on a period one orbit: i.e., one sets $y_{2}=y_{0}$ and $t_{2}-t_{0}=2 \pi / \omega$ to obtain

$$
\bar{D}=\mathrm{e}^{-4 \pi \alpha / \omega}, \bar{T}=\mathrm{e}^{-2 \pi \alpha / \omega}\left[-\mathrm{s}_{+} \mathbf{S}_{-}\left\{\frac{\left(\Omega_{+}^{2}+\Omega_{-}^{2}\right)}{\Omega_{+} \Omega_{-}}\right\}+2 c_{+} c_{-}\right] .
$$

$\bar{D}$ and $\bar{T}$ determine the eigenvalues $\lambda_{i}$ of DP evaluated on the period one point via the expression

$$
\lambda_{1,2}=\frac{1}{2}\left(\bar{T} \pm \sqrt{\bar{T}^{2}-4 \bar{D}}\right) .
$$

These eigenvalues determine the stability of the period one point [21, 22]. If $\lambda_{1}$ and $\lambda_{2}$ lie inside the unit circle $C$, then the fixed point is stable, while if either one lies outside $C$ the fixed point is unstable. As one varies the system parameters, eigenvalues may pass through the unit circle, at which point a bifurcation occurs. Note that since $\lambda_{1} \lambda_{2}=\bar{D}$ and $\bar{D}<1$, the only possible way for an eigenvalue to pass through $C$ is either through +1 or through -1: i.e., no Hopf bifurcations to doubly periodic motions can occur [21]. 
The condition that $\lambda= \pm 1$, from equation (36), is

$$
\bar{D} \mp \bar{T}+1=0 .
$$

Using equations (34) and (35) in (37) one obtains

$$
\mathrm{e}^{-4 \pi \alpha / \omega} \mp 2 \mathrm{e}^{-2 \pi \alpha / \omega}\left[-\hat{\Omega}_{\mathrm{S}_{+} \mathrm{S}_{-}}+\mathrm{c}_{+} \mathrm{C}_{-}\right]+1=0,
$$

where $\hat{\Omega}=\frac{1}{2}\left\{\left(\Omega_{+}^{2}+\Omega_{-}^{2}\right) / \Omega_{+} \Omega_{-}\right\}$. This equation is quadratic in $\mathrm{e}^{-2 \pi \alpha / \omega}$ and, in order for $\alpha$ and $\omega$ to be real and positive, it must have real roots between 0 and 1 . This gives a necessary condition for a $(\lambda= \pm 1)$ bifurcation to occur:

$$
\pm\left(\mathbf{c}_{+} \mathbf{c}_{-}-\hat{\Omega}_{\left.\mathbf{s}_{+} \mathbf{s}_{-}\right)}>1\right. \text {. }
$$

Up to this point no assumptions or approximations have been made.

Equation (38) for the bifurcation condition can now be examined. Note that the equation depends on the system parameters $\alpha, \omega$, and $\tilde{\omega}$ while it is apparently independent of $x_{0}$ and $\beta$. However, it is also important to note that equation (38) contains the terms $s_{ \pm}$and $c_{ \pm}$which depend on the times of flight $\left(t_{1}-t_{0}\right)$ and $\left(t_{2}-t_{1}\right)$, and hence implicitly upon $x_{0}$ and $\beta$ as well as the other parameters. This presents a difficulty, since the times of flight are not known (although $\left(t_{2}-t_{0}\right)=2 \pi / \omega$ is known). The values of $t_{0}, t_{1}$, and $t_{2}$ are determined by the system parameters, but through the unknown functions $f$ and $g$ (equation (20)).

We now make an important assumption regarding these times of flight based on observations of numerical simulations of the system. This assumption appears valid only for $x_{0}$ small compared with the maximum amplitude of the period one orbit. It was observed that the times of flight for a period one orbit are distributed approximately as in the unforced problem. (Thus, the orbit spends less time on the side of $x_{0}$ with the greater stiffness than it does on the side with smaller stiffness.) We assume, based on these observations, that for $x_{0}$ small,

$$
\left(t_{1}-t_{0}\right)=\pi \Omega_{n} / \omega \Omega_{+} \text {and }\left(t_{2}-t_{1}\right)=\pi \Omega_{n} / \omega \Omega_{-} .
$$

Using equations (40) one obtains

$$
\begin{gathered}
\Omega_{+}\left(t_{1}-t_{0}\right)=\Omega_{-}\left(t_{2}-t_{1}\right)=\pi \Omega_{n} / \omega \text { and } \\
\mathrm{s}_{+}=\mathrm{s}_{-}=\sin \left(\pi \Omega_{n} / \omega\right) \stackrel{\text { def }}{=} \mathrm{s}, \quad \mathrm{c}_{+}=\mathrm{c}_{-}=\cos \left(\pi \Omega_{n} / \omega\right) \stackrel{\text { def }}{=} \mathrm{c} .
\end{gathered}
$$

Using equations (41) in equation (38) then gives

$$
\mathrm{e}^{-4 \pi \alpha / \omega} \mp 2 \mathrm{e}^{-2 \pi \alpha / \omega}\left[-\hat{\Omega} \mathrm{s}^{2}+\mathrm{c}^{2}\right]+1=0,
$$

an expression involving only $\alpha, \omega$, and $\tilde{\omega}$. Also, condition (39) becomes

$$
\pm\left(\mathrm{c}^{2}-\hat{\Omega} s^{2}\right)>1 \text {. }
$$

One therefore sees immediately that, since $0 \leqslant c^{2} \leqslant 1$ and $\hat{\Omega} \mathrm{s}^{2} \geqslant 0$, only the $\lambda=-1$ bifurcation is possible (under our assumption regarding the times of flight). This is a flip bifurcation [21], in which a period two orbit branches out from the bifurcation point, generally in one of two ways. Figure 5(a) shows a supercritical flip bifurcation in which a stable period two orbit appears and the period one orbit becomes unstable. Figure 5(b) shows a subcritical flip bifurcation in which an unstable period two orbit merges with the stable period one orbit and an unstable period one orbit remains. Higher order terms must be computed to determine which type of bifurcation occurs. 


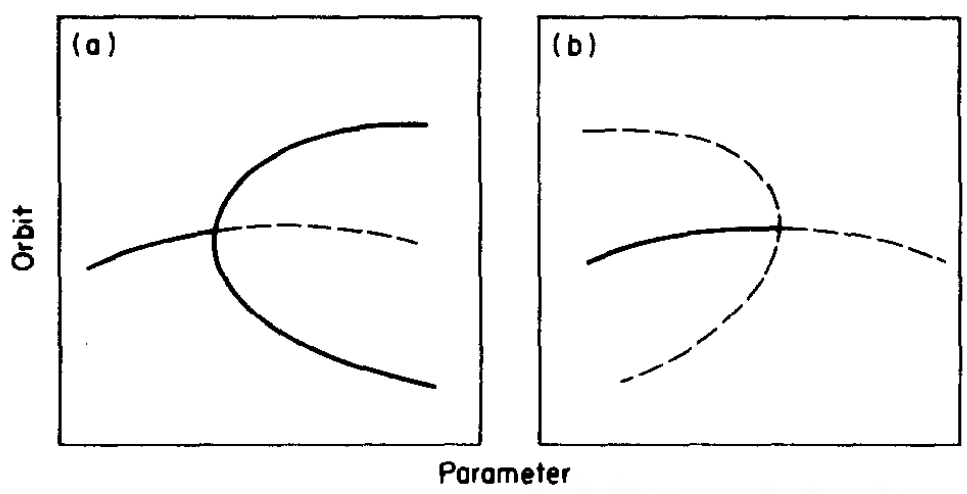

Figure 5. A (a) supercritical and (b) subcritical flip bifurcation.

The bifurcation condition (42) for the $\lambda=-1$ case can be solved for $\mathrm{e}^{-2 \pi \alpha / \omega}$ and then for $\alpha / \omega$ to obtain

$$
\alpha / \omega=-(1 / 2 \pi) \ln \left[\left(\hat{\Omega} \mathrm{s}^{2}-\mathrm{c}^{2}\right)-\sqrt{\left(\hat{\Omega} \mathrm{s}^{2}-\mathrm{c}^{2}\right)^{2}-1}\right] .
$$

We have taken the "minus" root of the quadratic since it is the one that gives $\alpha, \omega>0$. Note that $\alpha$ and $\omega$ appear in both sides of this equation. It may be solved numerically by choosing $\tilde{\omega}$ and $\omega$ and solving for the corresponding bifurcation value of $\alpha$ by a simple root finding method. This was done for several values of $\tilde{\omega}$ over a range of $\omega$ and the results are shown in Figure 6 as curves in $\left(\alpha, \omega / \omega_{n}\right)$ space for several values of

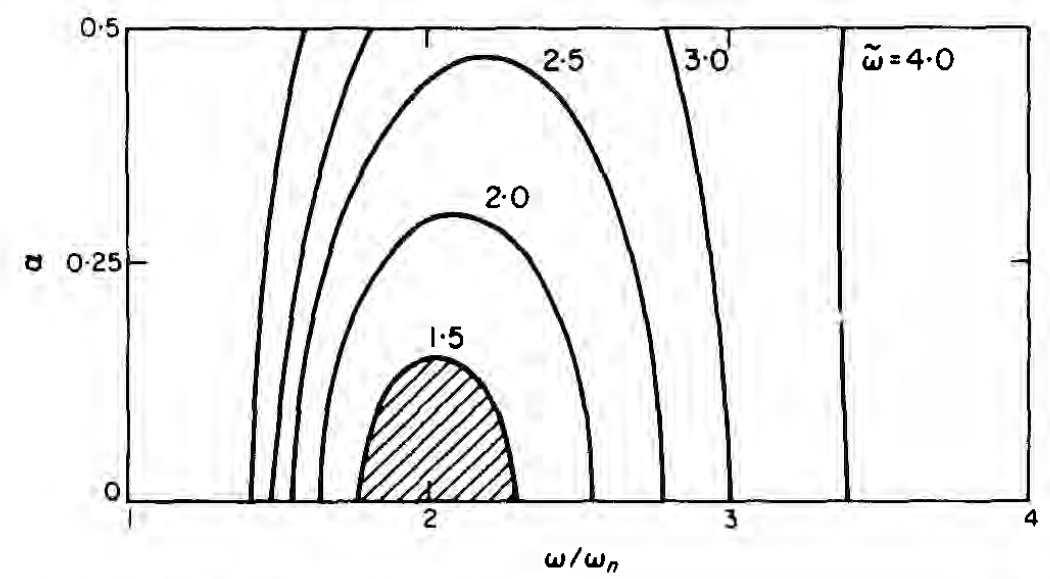

Figure 6. $\lambda=-1$ bifurcation curves for finite stiffness ratio, shown in $\left(\alpha, \omega / \omega_{n}\right)$ space for values of $\tilde{\omega}$.

$\tilde{\omega}$. For $\tilde{\omega}=1.5$ the region in which the period one orbit is unstable is shaded. Note that as $\tilde{\omega} \rightarrow 1$, these curves collapse around the point $\alpha=0, \omega / \omega_{n}=2$. This agrees with the known result that $\lambda=-1$ for the linear oscillator only at that point. In order to determine the type of flip bifurcation which occurs as a bifurcation curve is crossed, we now consider a special case.

Making the assumption that the forcing frequency $\omega$ is almost twice the value of the natural frequency $\Omega_{n}$, i.e., $\omega=2 \Omega_{n}+\varepsilon,|\varepsilon| \ll 1$, one obtains

$$
\mathrm{s}=\sin \left(\pi \Omega_{n} / \omega\right)=1+O\left(\varepsilon^{2}\right) \text { and } \mathrm{c}=\cos \left(\pi \Omega_{n} / \omega\right)=O(\varepsilon) .
$$

This, along with the small damping assumption $\alpha \ll 1$, reduces equation (44) to the simple expression

$$
\alpha / \omega=|(1 / 2 \pi) \ln (\tilde{\omega})|+O\left(\alpha^{2}, \varepsilon^{2}\right)
$$


A computation using center manifold methods, outlined in the Appendix, shows that this bifurcation is supercritical. Therefore a stable period two orbit exists just below the bifurcation curves shown in Figure 6.

In carrying out this analysis, we have assumed that a period one orbit exists. This follows from a simple continuation argument. When $\tilde{\omega}=1$, one has a linear oscillator and a unique (harmonic) $2 \pi / \omega$ periodic solution exists for $\omega \neq 1$. Moreover, if $\alpha>0$, this orbit is stable and will therefore continue to exist for $\tilde{\omega}$ close to one, since the map P has a stable period one point for $\tilde{\omega}=1$, at which (Id-DP) is invertible. Use of the implicit function theorem [23] then implies that $\mathbf{P}$ continues to have a period one point for $\tilde{\omega}$ near one. The only way in which this orbit can cease to exist, as $\tilde{\omega}$ increases, is by coalescence with another (unstable) period one motion [21] in which case an eigenvalue of DP evalued at the orbit would reach +1 . But we have shown that (under our time of flight assumption) this cannot occur. Therefore a period one orbit exists for all $\tilde{\omega}$. The numerical results shown below, and analysis of the $\tilde{\omega} \rightarrow \infty$ limit, bear this out.

The times of flight assumption (40) is approximately true only for $x_{0}$ small compared to the maximum amplitude of the period one orbit. For $x_{0} \neq 0$, as $\omega$ is increased, this orbit will shrink and eventually become simply the steady state solution of equation (2). The value of $\omega$ at which this occurs can be determined by setting $x_{0}$ equal to the maximum amplitude of that steady solution and solving the resulting equation for $\omega$.

For large damping, $\alpha>\tilde{\omega}>1$, one must use the overdamped solutions of equations (2) and (3). A calculation of DP in that case proceeds just as in the undamped $(\alpha<1<\tilde{\omega})$ case. When the damping becomes very large, $\alpha \gg \tilde{\omega}$, and $x_{0}$ is very small $\left(x_{0}=O\left(1 / \alpha^{2}\right)\right)$, it can be shown that

$$
\bar{T}=1-(\pi / 2 \alpha \omega)\left(1+\tilde{\omega}^{2}\right)+O\left(1 / \alpha^{2}\right),
$$

while $\bar{D}$ is exponentially small. Thus the eigenvalues are, from equation (36),

$$
\lambda_{1}=0 \text { and } \lambda_{2}=\bar{T},
$$

up to exponentially small terms. One concludes that no bifurcations occur as $\alpha$ is increased to large values. Here we have assumed that the times of flight are equal and are both $\pi / \omega$. This is so since one expects the solution leaving $x_{0}$ to be very close to the steady state solution of the appropriate equation (2) or (3). These steady solutions are of size $O(1 / \alpha)$ and both are approximately $90^{\circ}$ out of phase with the force. Thus their phases match well at $x=x_{0}$. Their velocities also match well (to $O\left(1 / \alpha^{2}\right)$ ), due to the large damping. Since one expects the period one orbit to lie near these solutions on either side of $x=x_{0}$, the modified time of flight assumption seems reasonable.

\subsection{GENERAL CASE, DIGITAL SIMULATION}

The matching of solutions (4) and (5) described previously can be done easily on a computer. Initial conditions $\left(t_{0}, y_{0}\right)$ are set in $\Sigma$ and the equation $x_{+}\left(t ; t_{0}, y_{0}\right)$ then determines the motion until $x(t)$ reaches $x_{0}$ again. The computer then solves for the $x_{0}$ crossing time, $t_{1}$, using a simple Newton-Raphson method [24] on equation (18); $t_{1}$ is used to compute the velocity from equation (19). The new time and velocity are used as initial conditions in $x_{-}\left(t ; t_{1}, y_{1}\right)$, which gives the motion exactly until $x=x_{0}$ once more. The procedure is repeated at length to obtain a solution of equation (1). From this global time solution one easily obtains the iterates of the mapping $\mathbf{P}$ by recording the values of $(t, y)$ at each $x_{0}$ crossing for which $y>0$. Note that this solution is considerably more accurate than the usual numerical solutions of ordinary differential equations [24], the only approximations being made at the $x_{0}$ crossing points, which can be easily computed 
to high precision. The analytical results from the previous section are verified by using this simulation.

First one can verify equation (46), the simplified bifurcation condition at $\omega \simeq 2 \Omega_{n}$ and $\alpha \ll 1$. Taking values of $\tilde{\omega}$ of $1.2,1.4,1.6$ and 1.8 one sets $\omega=4 \omega /(1+\tilde{\omega})=2 \omega_{n}=$ $2 \Omega_{n}+O\left(\alpha^{2}\right)$. The damping is then varied in small increments and the steady state solution observed. In this way one finds the actual bifurcation value to moderate precision. These values are then checked with those computed from equation (46). Table 1 and Figure 7 show good agreement between the predicted and the actual bifurcation points. The times of flight are all found to be within $5 \%$ of values assumed on the basis of equation (40) with $\alpha \ll 1$.

TABLE 1

Bifurcation values

\begin{tabular}{|c|c|c|c|c|c|}
\hline$\tilde{\boldsymbol{\omega}}$ & $\omega=2 \omega_{n}$ & $\begin{array}{c}\alpha_{\mathrm{cr}} \text { from } \\
\text { equation }(46)\end{array}$ & $\begin{array}{c}\alpha_{\mathrm{cr}} \text { from digital } \\
\text { simulation }\end{array}$ & $\begin{array}{c}\bar{t}, \bar{y}(\text { all are } \\
\pm 0 \cdot 003)\end{array}$ & $a$ \\
\hline $1 \cdot 2$ & $2 \cdot 182$ & 0.063 & $0.066 \pm 0.002$ & $0.738,0.605$ & 0.07 \\
\hline $1 \cdot 4$ & $2 \cdot 333$ & $0 \cdot 125$ & $0 \cdot 128 \pm 0.002$ & $0.708,0.554$ & 0.16 \\
\hline $1 \cdot 6$ & $2 \cdot 462$ & 0.184 & $0 \cdot 190 \pm 0 \cdot 002$ & $0.680,0.512$ & $0 \cdot 26$ \\
\hline $1 \cdot 8$ & $2 \cdot 571$ & 0.241 & $0 \cdot 250 \pm 0.002$ & $0.660,0.475$ & 0.37 \\
\hline
\end{tabular}

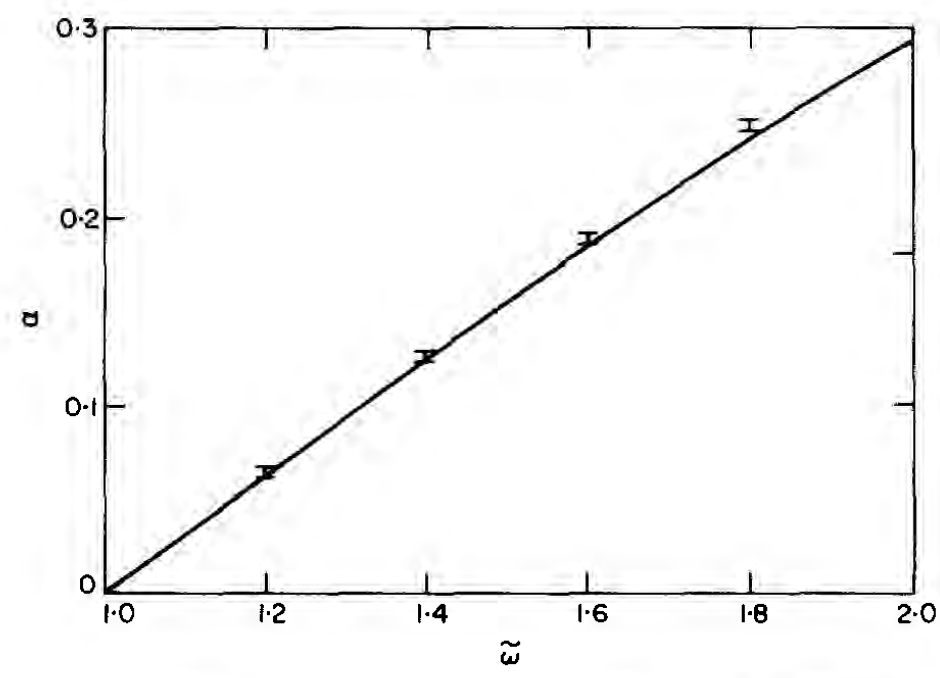

Figure 7. Bifurcation values of $\alpha v$ s. $\tilde{\omega}$ for $x_{0}=0, \beta=1$ and $\omega=2 \omega_{n}$. Solid curve is theory (equation (46)). Error bars I from digital simulation at $\tilde{\omega}=1 \cdot 2,1 \cdot 4,1 \cdot 6$ and 1.8 .

As $\alpha$ is lowered below the period doubling value, for parameter values $\tilde{\omega}<1 \cdot 5, x_{0}=0$, $\omega \simeq 2 \Omega_{n}$, one finds no further bifurcations. This is in contrast with the period doubling cascades found in many non-linear systems. When $\tilde{\omega}$ becomes large, however, other bifurcations can occur. The limit $\tilde{\omega} \rightarrow \infty$ will subsequently be studied in detail.

We now present an example using the more general bifurcation condition (44). Setting $\tilde{\omega}=4$ (a stiffness ratio of 16 ) and $\alpha=0 \cdot 125$, we solved equation (44) numerically to determine $\omega_{\text {bif }} \cong 2 \cdot 26$. Figures $8(a)$ and (b) show' that the bifurcation actually occurs between $\omega=2.40$ and $\omega=2.42$. The error is due to the fact that the actual times of flight for the period one orbit near the bifurcation value differ from the assumed values by $6 \%$. 


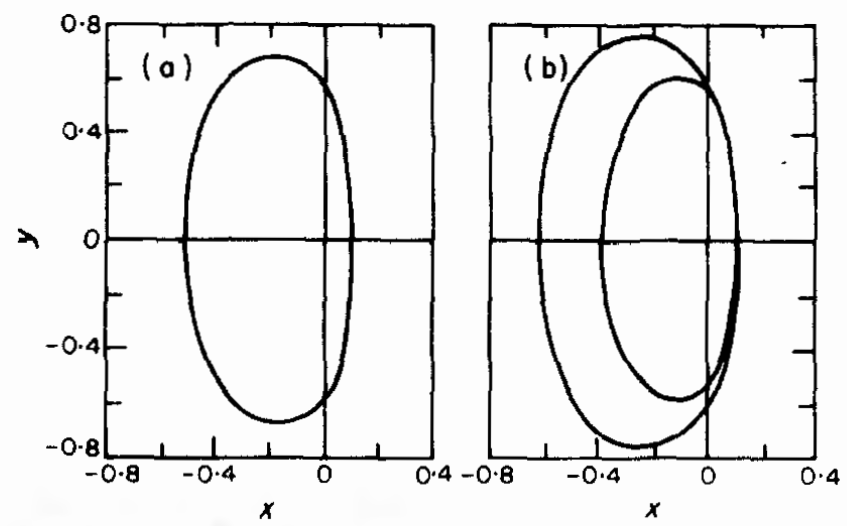

Figure 8. (a) Stable period one orbit at $\omega=2 \cdot 40, \alpha=0 \cdot 125, x_{0}=0, \beta=1$, and $\tilde{\omega}=4$, from digital simulation; (b) stable period two orbit at $\omega=2 \cdot 42, \alpha=0 \cdot 125, \beta=1, x_{0}=0$, and $\hat{\omega}=4$, from digital simulation. Both in projected phase plane $(x, y)$.

By using digital simulations, other subharmonic orbits are also found to exist. Figures 9(a) and (b) show the coexistence of single impact stable period one and stable period three orbits for $x_{0}=0, \alpha=0 \cdot 026, \tilde{\omega}=\sqrt{2}$ and $\omega=3 \cdot 5$. We conjecture that the period three orbit appeared in a saddle-node bifurcation [21] and that an unstable period three orbit also exists at these parameter values. Analysis of this bifurcation is much more difficult since no reasonable assumption regarding the times of flight can be made and higher iterates of the mapping (multiple impacts) appear to be involved.

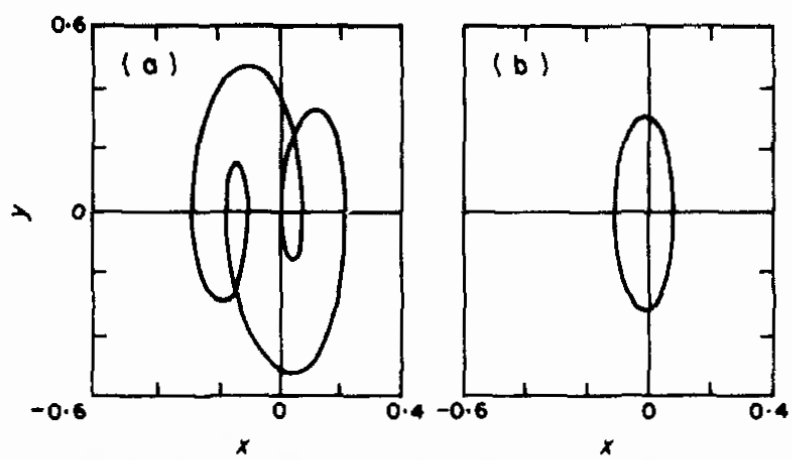

Figure 9. Coexistence of stable period three and stable period one at $\tilde{\omega}=\sqrt{2}, \alpha=0.026, \beta=1, x_{0}=0$ and $\omega=3.5$. (a) Period 3; (b) period 1. Both in projected phase plane $(x, y)$.

\subsection{THE IMPACT LIMTT, ANALYSIS}

In the impact limit an important simplification occurs, since the time of flight during the impact is taken to be zero. This allows more analysis to be done on periodic orbits. We also rescale: $x \rightarrow \beta x$, and take unit forcing amplitude.

The return map for the impact oscillator is very similar to the one for the general system. From points 0 to 1 in Figure 4 one uses the impact limit, i.e.,

$$
t_{1}=t_{0} \text {, and } y_{1}=-r y_{0} \text {. }
$$

From points 1 to 2 one uses the same mapping as described in the general case. Thus the mapping $\mathbf{P}$ still cannot be written down explicitly. As before, however, one can compute DP analytically. Moreover, in this limiting case one can compute periodic points corresponding to single impact, period $\boldsymbol{n}$ orbits directly. Such orbits correspond to those motions which strike the wall (the very stiff spring $k_{2}$ ) and then remain in $x<x_{0}$ for 
exactly $2 \pi n / \omega$ in time and then strike the wall again with the same velocity as the previous impact. The conditions for the existence of such an orbit are

$$
t_{2}-t_{0}=2 \pi n / \omega, \quad y_{2}=y_{0}=-y_{1} / r, \quad \text { with } y_{0}>0 \text {. }
$$

These two conditions allow one to compute the period $n$ point $(\bar{t}, \bar{y})$. First one writes equation (7), using equations (5), (50) and (51), to obtain

$$
0=-x_{0} \Gamma_{-}+\Lambda y_{1}+\mathrm{s}_{1}\left(\delta \Gamma_{-}+\Lambda \gamma \omega\right)+\mathrm{c}_{1}\left(\gamma \Gamma_{-}-\Lambda \delta \omega\right),
$$

where we have dropped the minus subscripts on $\gamma, \delta$, and $\Omega$ and where $\Gamma_{ \pm}=1-E \mathrm{c} \pm \alpha \Lambda$, $\Lambda=E \mathrm{~s} / \Omega, E=\mathrm{e}^{-2 \pi n \alpha / \omega}, \mathrm{s}=\sin (2 \pi n \Omega / \omega)$, and $\mathrm{c}=\cos (2 \pi n \Omega / \omega)$. Next one writes equation (51) using equation (50) and the time derivative of equation (5) to obtain

$$
0=y_{1}\left(-1-r+r \Gamma_{+}\right)+\Lambda r x_{0}+s_{1} r\left(\gamma \omega \Gamma_{+}-\Lambda \delta\right)-c_{1} r\left(\delta \omega \Gamma_{+}+\Lambda \gamma\right)
$$

Since $y_{1}$ appears in a linear manner in both equations (52) and (53), it may be eliminated to obtain a single equation involving only $t_{1}$ as an unknown (in the terms $c_{1}=\cos \left(\omega t_{1}\right)$ and $\left.s_{1}=\sin \left(\omega t_{1}\right)\right)$ :

$$
\begin{gathered}
0=\left(x_{0} / \Lambda\right)\left[r \Lambda^{2}-\Gamma_{-} \psi\right]+\mathrm{s}_{1}\left[r \gamma \omega \Gamma_{+}-\Lambda \delta r+(\psi / \Lambda)\left(\delta \Gamma_{-}+\Lambda \gamma \omega\right)\right] \\
+\mathrm{c}_{1}\left[-r \delta \omega \Gamma_{+}-\Lambda \gamma r+(\psi / \Lambda)\left(\gamma \Gamma_{-}-\Lambda \delta \omega\right)\right],
\end{gathered}
$$

where $\psi=1+r-r \Gamma_{+}$. Straightforward association of terms allows this equation to be written as

$$
0=X+\mathrm{s}_{1} Y+\mathrm{c}_{1} Z
$$

which has a solution

$$
\bar{t}_{1}=(1 / \omega)[\arctan (Y / Z)+\arccos (-X / W)],
$$

where $W=\sqrt{Y^{2}+Z^{2}}$. This expression gives the time (i.e., forcing phase) at impact on the period $n$ orbit. The velocity just after impact $\bar{y}_{1}$ is then easily computed by using either equation (52) or equation (53).

It is important to note that a solution obtained as described above only satisfies $x\left(t_{1}\right)=x_{0}$ and $\dot{x}\left(t_{1}\right)=-r \dot{x}\left(t_{2}\right)$. If the value of $\bar{y}_{1}=\dot{x}\left(t_{1}\right)$ is positive, then the solution corresponds to a non-physical, or "penetrating" orbit [13]. The orbits for $\bar{y}_{1}$ negative must also be checked since nowhere has one been assured that the desired $x_{0}$ crossing is the first on the orbit. In fact, the above conditions can be satisfied after several $x_{0}$ crossings, for some parameter values. Care must be taken to determine which of these orbits are physically possible.

Knowing the periodic point, one can now compute its stability. As before, one breaks the calculation of DP into two parts, from point 0 to 1 and from point 1 to point 2 . Here

$$
\left[\frac{\partial\left(t_{1}, y_{1}\right)}{\partial\left(t_{0}, y_{0}\right)}\right]=\left[\begin{array}{cc}
1 & 0 \\
0 & -r
\end{array}\right], \text { and } \mathbf{D P}=\left[\frac{\partial\left(t_{2}, y_{2}\right)}{\partial\left(t_{1}, y_{1}\right)}\right]\left[\begin{array}{cc}
1 & 0 \\
0 & -r
\end{array}\right] \text {, }
$$

where $\left[\partial\left(t_{2}, y_{2}\right) / \partial\left(t_{1}, y_{1}\right)\right]$ was derived above in the analysis of the finite stiffness case. From this calculation one finds that DP has determinant

and trace

$$
D=\left(-r y_{1} / y_{2}\right) \mathrm{e}^{-2 \alpha\left(t_{2}-t_{0}\right)},
$$

$$
T=\left[\mathrm{e}^{-\alpha\left(t_{2}-t_{0}\right)} / \Omega y_{2}\right]\left[\left(N_{1}+r N_{2}\right) \sin \left(\Omega\left(t_{2}-t_{0}\right)\right)+\Omega\left(y_{1}-r y_{2}\right) \cos \left(\Omega\left(t_{2}-t_{0}\right)\right)\right] .
$$

Evaluating on a period $n$ orbit gives

$$
\bar{D}=r^{2} E^{2} \text { and } \bar{T}=\left(E / \Omega \bar{y}_{0}\right)\left[(1+r)\left(\bar{c}_{0}+x_{0}\right) \mathrm{s}-2 r \bar{y}_{0} \Omega \mathrm{c}\right],
$$


where $\bar{y}_{0}=-\bar{y}_{1} / r$ and $\bar{c}_{0}=\cos \left(\omega \bar{t}_{0}\right)=\cos \left(\omega \bar{t}_{1}\right)$. Using equation (36) one can determine the eigenvalues and thus the stability of the period $n$ point. The above analysis is quite general.

In order to study this impact system in more detail we shall consider a special case in which the equations simplify significantly: we shall take $\alpha=0$ and $x_{0}=0$ in what follows. This case corresponds to the wall being at the origin and there being no damping in the $x<0$ motion. Note that the coefficient of restitution continues to provide an energy loss mechanism.

Proceeding exactly as in the general case, one obtains a simplified version of equation (56):

$$
\bar{t}_{1}=(1 / \omega) \arctan [+(1-r)(1-c) /\{-(1+r) \omega s\}] .
$$

Solving for $\bar{y}_{1}$ yields

$$
\bar{y}_{1}=2 \omega r(1-c) /\left(1-\omega^{2}\right)\left\{[(1-c)(1-r)]^{2}+[\omega s(1+r)]^{2}\right\}^{1 / 2} .
$$

Note that requiring $\bar{y}_{1}<0$ implies that $\omega>1$; thus these period $n$ single impact orbits do not exist for forcing frequencies below $\omega=1$. The determinant and trace on this orbit simplify to

$$
\bar{D}=r^{2} \quad \text { and } \quad \bar{T}=\left[\mathrm{s}^{2}(1+r)^{2}\left(1-\omega^{2}\right)-4 r \mathrm{c}(1-\mathrm{c})\right] / 2(1-\mathrm{c}) \text {, }
$$

respectively. It should be noted that in this case $\Omega=1, s=\sin (2 \pi n / \omega)$, and $c=$ $\cos (2 \pi n / \omega)$.

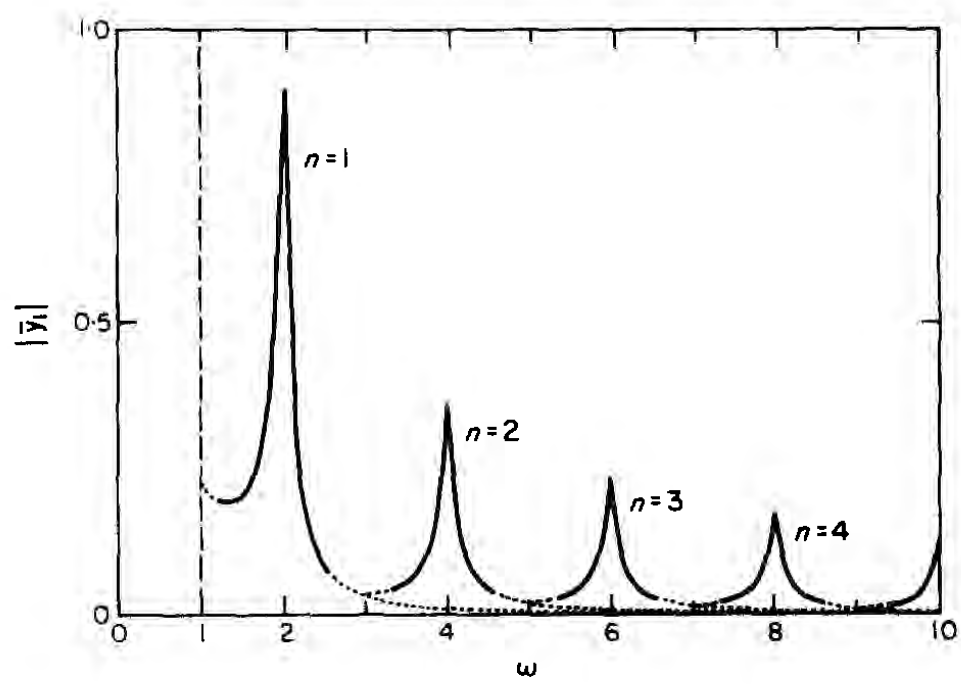

Figure 10. Resonance curve $\left|\bar{y}_{1}\right|$ vs. $\omega$ for simple impact period $n$ orbits. Solid portions correspond to stable orbits, dotted portions correspond to unstable orbits. Parameter values are $\alpha=0, x_{0}=0, r=0.8$.

Figure 10 shows a typical resonance curve for the impact oscillator with $r=0 \cdot 8$. The graph shows the magnitude of the velocity just after impact on period $n$ orbits versus forcing frequency $\omega$. The solid part of the curves represent stable orbits while the dashed parts represent unstable ones. Penetrating orbits were eliminated by checking enough points on the orbit to be sure that $t=t_{1}+2 \pi n / \omega$ was the first $x=0$ crossing time. These curves, produced analytically, show much similarity to those produced numerically by Thompson $[15,16]$. However, he also found multiple impact periodic orbits. 
As before, $\bar{D}<1$, so that only $\lambda= \pm 1$ bifurcations occur. Upon using equations (37) and (65) the $\lambda= \pm 1$ bifurcation condition, written as a quadratic in $r$, becomes

$$
r^{2} \mp 2 r\left(\frac{\mathrm{s}^{2}\left(1-\omega^{2}\right)-2 \mathrm{c}(1-\mathrm{c})}{\mathrm{s}^{2}\left(1-\omega^{2}\right)+2(1-c)}\right)+1=0 .
$$

The requirement that this equation have a root between 0 and 1 shows that only the $\lambda=-1$ case can occur. (This equation should be compared with equation (42) in the finite stiffness case.) Here $r$ does not appear in the coefficients of the quadratic and the equation may be solved exactly for the bifurcation curves. The parameters in equation (66) are the forcing frequency $\omega$, the period of the orbit $n$, and, of course, the coefficient of restitution $r$. For each $n$ one can vary $\omega$ and record those roots of the quadratic which fall between 0 and 1 . In this way one constructs, for each $n$, a bifurcation curve in $r-\omega$ space. These curves are shown in Figure 11. A calculation, which is outlined in the Appendix, shows that the bifurcations are supercritical. Therefore one expects a stable, two impact, orbit of period $2 n$ to appear just outside both edges of each stable period $n$ region in Figure 11.

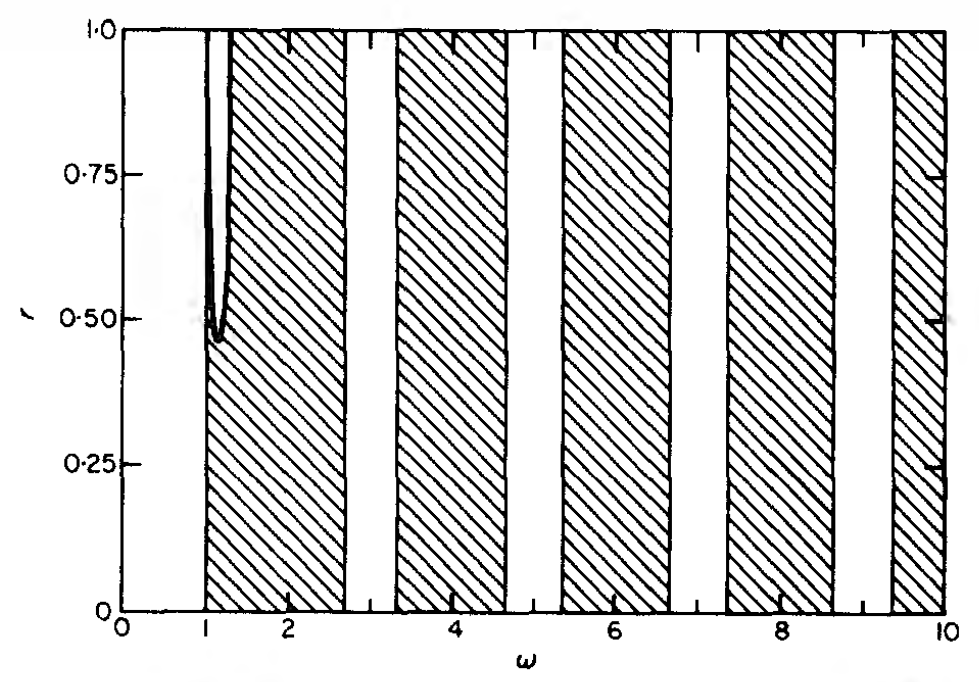

Figure 11. $\lambda=-1$ bifurcation curves of single impact orbits for $x_{0}=0, \beta=1$, and $\alpha=0$ in $r-w$ space. Shaded regions are stable.

The $n=2,3,4, \ldots$ regions of stable impact orbits appear to be nearly identical. They are approximately vertical strips centered at $\omega=2 n$. However, although the right side of the $n=1$ region is similar to those for $n>1$, the left side is much different. There exists a small region above $r \approx 0.45$ and just to the right of $\omega=1$ in which the period one single impact orbit is unstable. At certain parameter values in this region there coexist stable two impact period two orbits (resulting from the flip bifurcation) and stable three impact period two orbits, both found by using the digital simulation explained below. Note that as $\omega=1$ is approached from the right, the period one orbit is stable for all values of $r<1$ (the unstable regions meet at $\omega=1, r=1$ and for $r$ near 1 the stable region becomes very thin). As $\omega$ passes through 1 into $\omega<1$, period one orbits become no longer physically possible.

Other multiple impact periodic orbits also exist in this system [15]. However, the analysis of their existence, stability, and the bifurcations from them requires numerical or approximate techniques, since the times of flight between each impact are not known explicitly. We now describe such a digital simulation for this system. 


\subsection{THE IMPACT LIMIT, DIGITAL SIMULATION}

The digital computer simulation of the impact oscillator is very similar to that for the finite stiffness case. One composes the linear solution (5) for $x<x_{0}$ with the impact relationship (49) in a straightforward manner. In this case each iterate of $\mathbf{P}$ requires the solution of one (instead of the previous two) transcendental equation, for the time of impact with the wall. This digital simulation is the means by which we obtained the following results for the impact oscillator, including those in the following section, in which chaotic motions were observed.
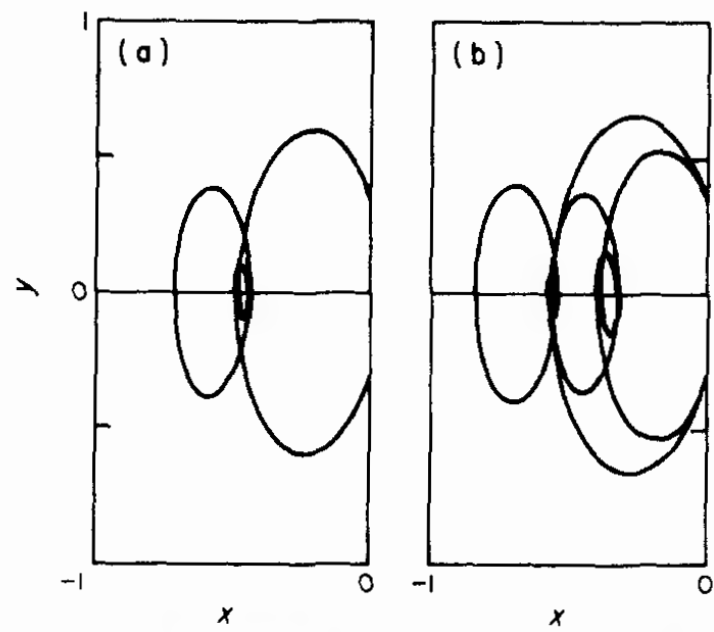

Figure 12. Period doubling of impact oscillator; $r=0 \cdot 8, x_{0}=0, \beta=1, \alpha=0$ and $n=3$. (a) Stable period three orbit at $\omega=5 \cdot 37$; (b) stable period six orbit at $\omega=5 \cdot 35$. Both in projected phase plane $(x, y)$.

Here we present two typical periodic motions of this system. Figure 12(a) shows a stable period 3 orbit of single impact type found by the above analysis and generated by using the digital simulation. The parameter values are $r=0.8$ and $\omega=6.63\left(x_{0}=0\right.$ along with $\alpha=0$ ). These values near the bifurcation point $r=0.8, \omega \cong 6.635$ determined from equation (66). Figure 12(b) shows a two impact period six orbit at $r=0 \cdot 8, \omega=6 \cdot 65$. This orbit appeared at the flip bifurcation. The period three orbit is of course still present, but is now unstable.

We next turn our attention to further bifurcations which cannot be studied analytically but require the use of the digital simulation.

\section{FURTHER PERIOD DOUBLINGS, HORSESHOES AND STRANGE ATTRACTORS}

\subsection{CASCADES OF PERIOD DOUBLINGS AND OTHER TRANSITIONS}

So far we have been able to find analytically single impact, period $n$ motions and the bifurcations from them to period $2 n$ double impact motions. The regions in Figure 11 which have no stable single impact orbits will now be examined in greater detail. The following results should be compared with those of Thompson $[15,16]$.

In Figure 10 (with $r=0.8$ ) the region between the stable $n=1$ orbit and the stable $n=2$ orbit lies in the range $\omega \cong 2 \cdot 6533$ to $\omega \cong 3 \cdot 3535$. Figure 13 shows the results of digital simulations in that range. The maximum displacement between each impact is plotted against $\omega$. One sees that the period one orbit undergoes further period doublings as $\omega$ is increased. This cascade of period doublings is typical of many non-linear systems. Approximate bifurcation values of $\omega$ were recorded and checked against the general 


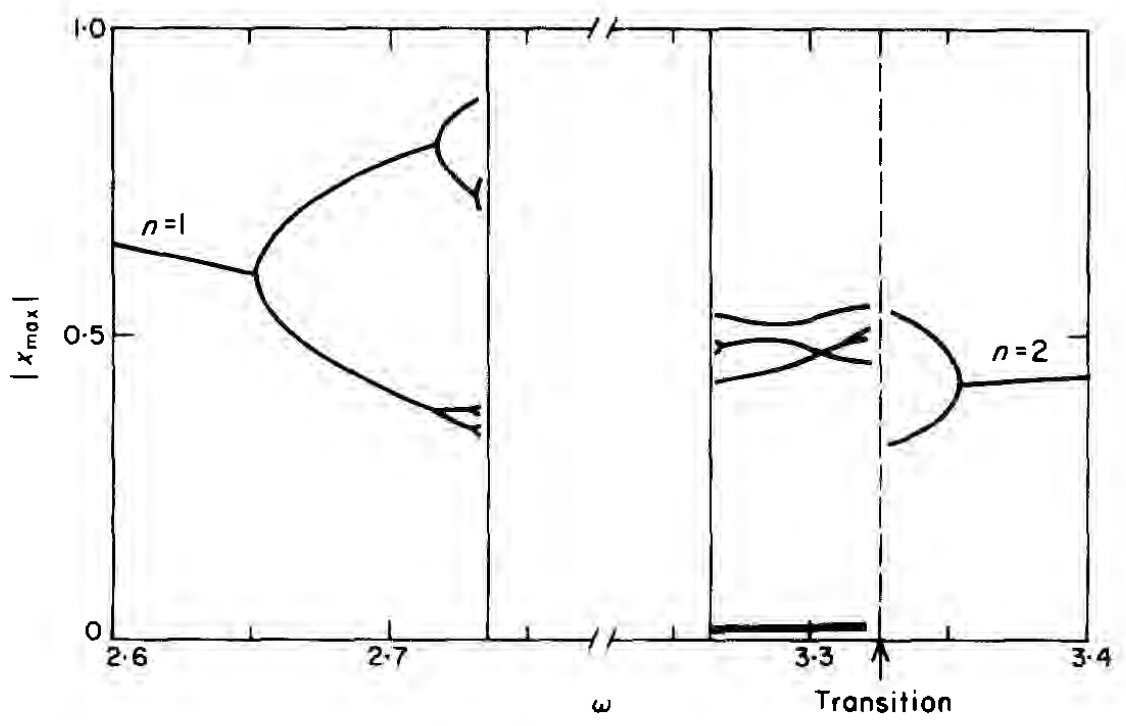

Figure 13. Bifurcation diagram between the stable $n=1$ and $n=2$ regions. Note period doubling sequence on the left and the transition and period doublings on the right. $\alpha=0, \beta=1, x_{0}=0, r=0 \cdot 8, \omega=2 \cdot 65$ to $3 \cdot 36$. Plot is of maximum $x$ values in between impacts, $x_{\text {MAX }} v s$. $\omega$.

asymptotic result due to Feigenbaum $[17,18]$,

$$
F_{\infty}=\lim _{j \rightarrow \infty} \frac{\omega_{j+2}-\omega_{j+1}}{\omega_{j+1}-\omega_{j}} \stackrel{\text { def }}{=} \lim _{j \rightarrow \infty} F_{i}=4 \cdot 669 \ldots,
$$

which one expects to hold for period doubling cascades in contracting $(\bar{D}<1)$ maps of this type [17]. Here $\omega_{j}$ is the bifurcation value from a period $2^{j}$ orbit to a period $2^{j+1}$ orbit.

Although the first such number produced, $F_{1}=4 \cdot 76 \pm 0.05$, is remarkably close to the expected limit, the succeeding two numbers appear to diverge and further numbers cannot be computed with sufficient accuracy (see Table 2). Nonetheless, there is clear digital evidence for an accumulation value, and orbits up to period 32 have been observed.

TABLE 2

Bifurcation values of $\omega$ for period $2^{j} \rightarrow 2^{j+1}$, and resulting Feigenbaum numbers $F_{j}[17,18]$

\begin{tabular}{ccl}
\hline$j$ & \multicolumn{1}{c}{$\omega_{j}$} & \multicolumn{1}{c}{$F_{j}$} \\
\hline 0 & \multicolumn{1}{c}{$2 \cdot 65335 \dagger$} & \\
1 & $2 \cdot 7158 \pm 0 \cdot 0001$ & \\
2 & $2 \cdot 72890 \pm 0.00003$ & $4 \cdot 76 \pm 0 \cdot 05$ \\
3 & $2 \cdot 73131 \pm 0.00003$ & $5 \cdot 4 \pm 0 \cdot 2$ \\
4 & $2 \cdot 73175 \pm 0.00003$ & $5 \cdot 0 \pm 1 \cdot 0$ \\
\hline
\end{tabular}

$\dagger$ Known from equation (63).

Figure 14 shows some of the orbits in the period doubling sequence. Figure 14(a) shows a simple period one orbit in the projected $(x, y)$ phase space at $\omega=2 \cdot 64$. Figure 14(b) shows the same orbit as a fixed point in $\Sigma$ (note that we have taken the variable $t$ modulo $2 \pi / \omega)$. Figure 14(c) shows the period 2 orbit at $\omega=2 \cdot 69$, and Figure 14(d) shows, the orbit in $\Sigma$. The period doublings occur very rapidly with increase of $\omega$ as shown, by the period four orbit of Figures 14 (e) and (f) at $\omega=2.725$ and the period eight orbits shown in Figures $14(\mathrm{~g})$ and $(\mathrm{h})$ at $\omega=2.731$. 

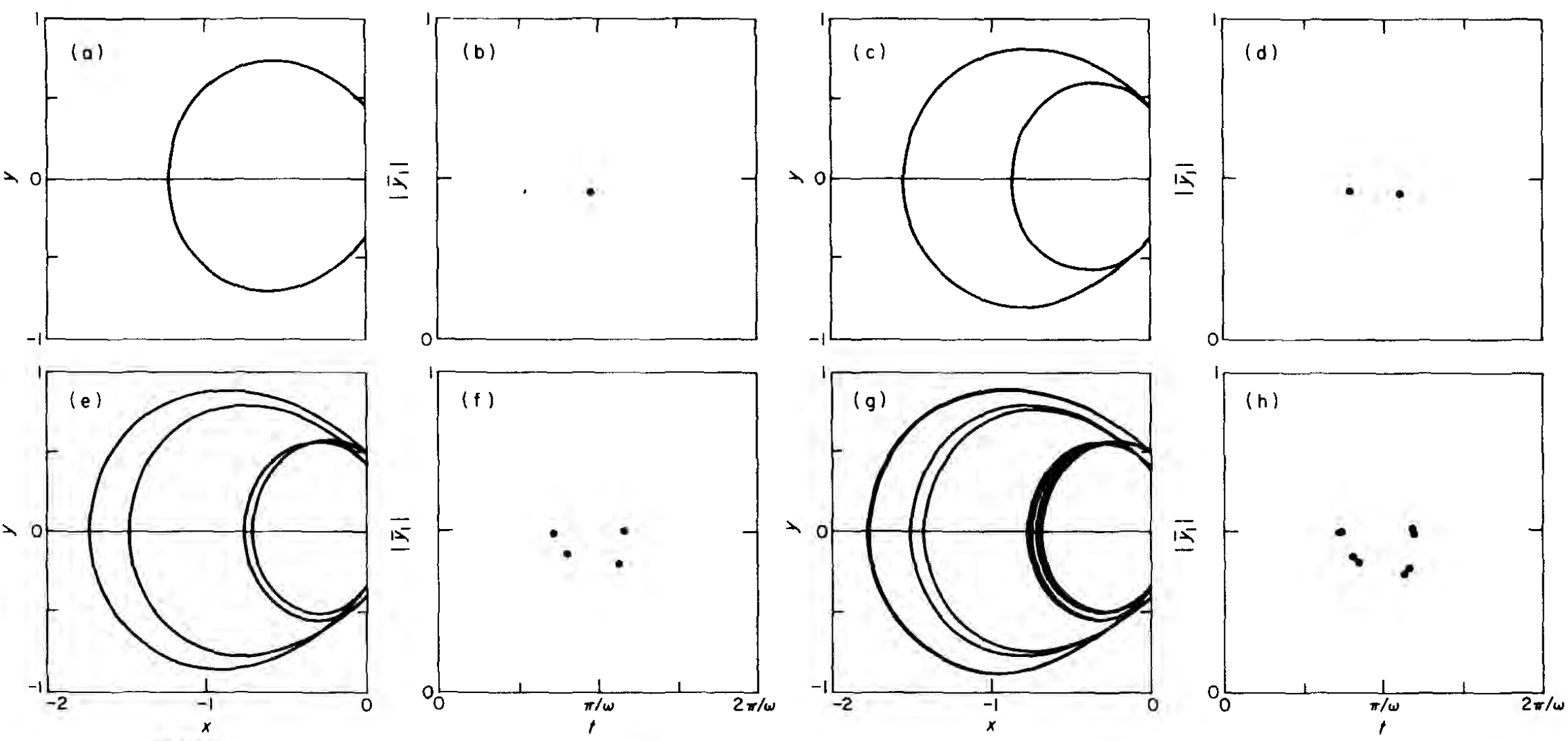

Figure 14. Period doubling sequence $\alpha=0, x_{0}=0, r=0 \cdot 8$. (a) Period 1 orbit in $(x, y), \omega=2 \cdot 640$; (b) period 1 orbit in $\Sigma$; (c) period 2 orbit in $(x, y)$ $\omega=2 \cdot 690$; (d) period 2 orbit in $\Sigma$; (e) period 4 orbit in $(x, y), \omega=2 \cdot 725$; (f) period 4 orbit in $\Sigma$; (g) period 8 orbit in $(x, y), \omega=2 \cdot 731$; (h) period 8 orbit in $\Sigma$. 
Equation (67) implies that the bifurcation values accumulate in a geometric series and hence at some finite value, $\omega_{\mathrm{ACC}}$, there is an orbit of "period $2^{\infty}$ ": i.e., a non-periodic orbit [17]. Based on the measured period doubling values of $\omega$ one can predict an approximate value for the accumulation point of $\omega_{\mathrm{ACC}} \cong 2 \cdot 732$.

However, the $n=2$ solution, after its first period doubling for decreasing $\omega$, can undergo a different type of transition. As $\omega$ is lowered and the period four solution develops, a point on the orbit becomes tangent at the origin and a degenerate impact occurs corresponding to a singular point for the mapping P. This will be discussed below. Thereafter, further bifurcations occur as shown in Figure 13 (including period doublings) until the solution appears to become non-periodic.

Thompson [16] has shown similar results for the case $r=1, \alpha \neq 0, x_{0}=0$. He studied the region between $n=4$ and the $n=5$ stable orbits using a similar digital simulation and found the same qualitative structure as shown in Figure 13. The "left" branch ( $n=1$ in the present case, $n=4$ in Thompson's [16]) period doubles in a straightforward manner. The "right" branch ( $n=2$ here and Thompson's $n=5$ [16]) doubles once to a double impact orbit. The orbit then passes through a singularity in the map and becomes a three impact orbit of the same period (period four $(T=8 \pi / \omega)$ in this study); see Figure 13 . Thereafter it appears to continue period doubling until non-periodic motions occur.

The transition from the period four double impact orbit to a period four triple impact orbit is not a bifurcation in the usual sense, as encountered in smooth maps [21]. As pointed out earlier, our map is not "onto" and consequently has discontinuities associated with orbits which leave $\Sigma$ and return at $x=y=0$. The transition referred to above occurs precisely when the double impact orbit passes through $x=y=0$. The nature of such transitions is determined by the particular map and its discontinuities and there is no general theory as in the case of "smooth" bifurcations [21]. However, clearly such transitions play an important role in the dynamics of piecewise linear systems and deserve further study.

The behavior in the unstable bands between $n=2$ and $n=3, n=3$, 4, etc., seems essentially the same as that in the first such band.

\subsection{THE EXISTENCE OF HORSESHOES}

In this section we take $r=1$ (the dissipationless case). Orbits are considered as iterates of the mapping $P$ and are shown as sequences of points in $\Sigma$. As in Figure 14, we take $t \bmod 2 \pi / \omega$, so that an $n$ impact periodic orbit appears as a fixed point of the $n$th iterate of $\mathbf{P}$, or as a cycle of $n$ periodic points of $\mathbf{P}$.

The location and stability of the period one point $(\bar{t}, \bar{y})$ is known exactly: within the range $\omega \approx 2.6533-3.3535$ it is an unstable fixed point of saddle type for the mapping P. We now examine the stable and unstable manifolds of $(\bar{t}, \bar{y})$.

The stable $\left(W^{s}\right)$ and unstable $\left(W^{u}\right)$ manifolds are defined as those sets of points which are respectively forward and backward asymptotic to $(\bar{t}, \bar{y})$ under iterates of $\mathbf{P}$ :

$$
W^{s}=\left\{\mathbf{x} \in \Sigma \mid \mathbf{P}^{n}(\mathbf{x}) \rightarrow(\bar{t}, \bar{y}) \text { as } n \rightarrow+\infty\right\}, \quad W^{u}=\left\{\mathbf{x} \in \Sigma \mid \mathbf{P}^{n}(\mathbf{x}) \rightarrow(\bar{t}, \bar{y}) \text { as } n \rightarrow-\infty\right\} .
$$

Since the map is piecewise smooth, but discontinuous, these sets are themselves disconnected, but we continue to refer to them as manifolds, as in the usual case of smooth mappings $[23,25]$.

The digital simulations indicate that the stable and unstable manifolds intersect, in the dissipationless case, for all values of $\omega$ for which $(\bar{t}, \bar{y})$ is unstable. Therefore a complicated invariant set, a Smale horseshoe, exists immediately after the bifurcation, but perhaps in some high iterate of $\mathbf{P}[25,26]$. 
A simple introduction to the horseshoe and its implications for chaos in iterated maps such as the present one can be found in reference [14] or [23]. More complete mathematical treatments have been given by Moser [26] and Guckenheimer and Holmes [25]. The main conclusion one can draw from the presence of horseshoes is that the map possesses a complicated invariant set $\boldsymbol{\Lambda}$ which contains (a) a countable infinity of unstable periodic orbits, including orbits of arbitrarily long periods; (b) an uncountable infinity of bounded, non-periodic orbits, and (c) a dense orbit. The horseshoe acts as a "chaotic saddle point", since while most orbits approaching it eventually leave its neighborhood, they do so in a manner which is extremely difficult to predict. We shall return to the effects and physical implications of such invariant sets in the next section.

For certain values of $\omega$ above the accumulation value $\omega_{\mathrm{ACC}}$, we can clearly demonstrate a horseshoe for the second iterate of $\mathbf{P}, \mathbf{P}^{2}$. For example, Figure 15 shows a plot, generated by the digital simulation, of $W^{s}$ and $W^{u}$ for $\omega=2 \cdot 8$. In this case $(r=1) \bar{t}=\pi / \omega$ and the reflectional symmetry about the line $\bar{t}=\pi / \omega$ should be noted. To see the horseshoe more clearly the area of interest is shown in Figure 16. The "primed" points

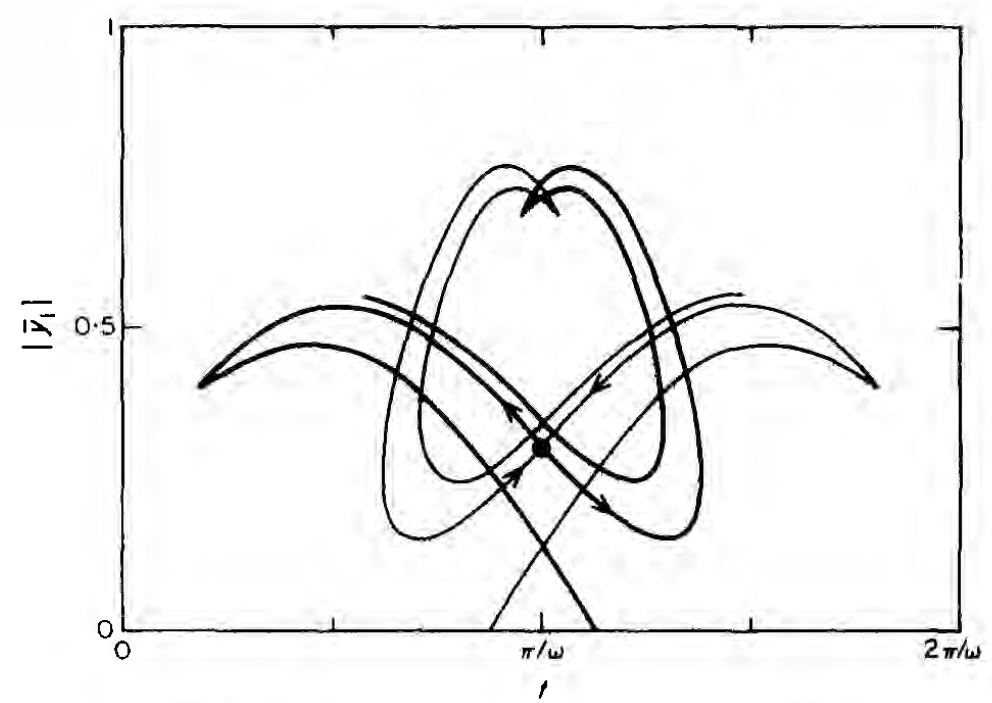

Figure 15. $W^{u}$ (heavy line) and $W^{s}$ (light line) in the dissipationless case $r=1, \alpha=0, x_{0}=0$, and $\omega=2 \cdot 8$. Note transversal intersections.

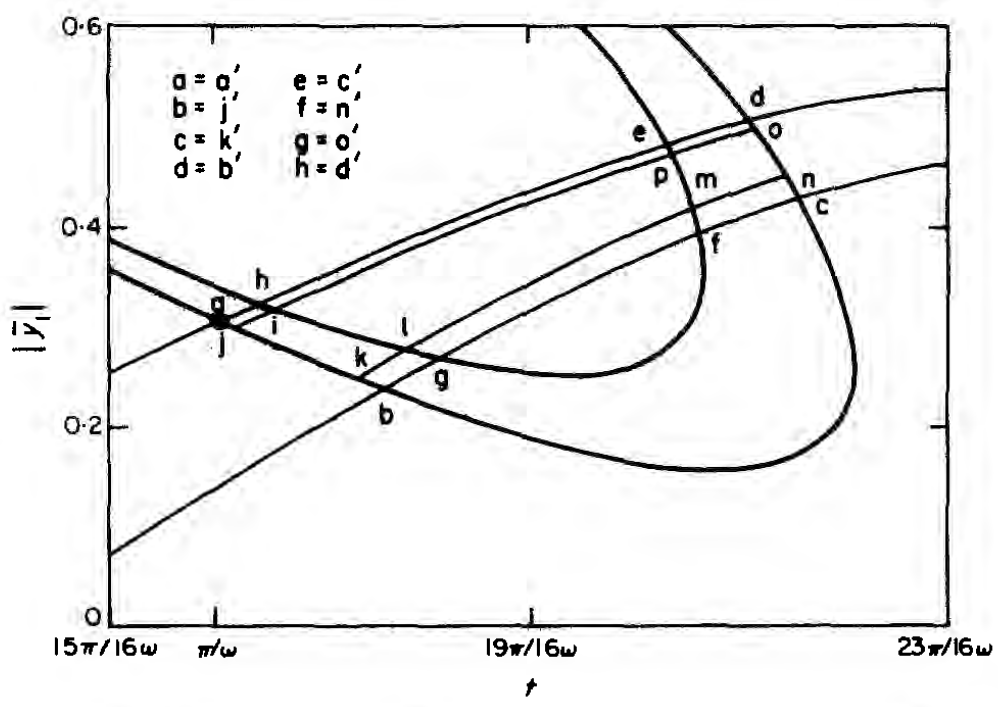

Figure 16. $W^{u}$ (heavy line) and $W^{3}$ (light line), as in Figure 15, showing region of interest and the horseshoePrimed points are images of unprimed points under $\mathbf{P}^{2}$. 
are the images of the unprimed points. The segments $\overline{o j}$ and $\overline{k n}$ are approximate preimages of segments $\overline{b g}$ and $\overline{f c}$. These segments were generated by approximating $\overline{b g}$ and $\overline{f c}$ by straight lines and iterating them twice under $\mathbf{P}^{-1}$ : i.e., once by $\mathbf{P}^{-2}$. $\left(\mathbf{P}^{-1}\right.$ is obtained in the digital simulation by simply running time backwards.) We define "horizontal" strips $H_{1}=a d o j$ and $H_{2}=k n c b$ along with "vertical" strips $V_{1}=a h g b$ and $V_{2}=e d c f$. Then by considering the images of $H_{1}$ and $H_{2}$ under $\mathbf{P}^{2}$, we see that

$$
\mathbf{P}^{2}\left(H_{i}\right)=V_{i}, \quad i=1,2,
$$

and thus we have a topological horseshoe [25].

The invariant set $\Lambda$ of points which never leave the two strips $H_{i}$ under forward or backward iteration of $\mathbf{P}^{2}$ is obtained by intersection of all the images

$$
\Lambda=\bigcap_{n=-\infty}^{\infty}\left(\mathbf{P}^{2}\right)^{n}\left(H_{1} \cup H_{2}\right)
$$

It can be shown to be a Cantor set with the properties outlined earlier (cf. reference [14]). The hyperbolicity and persistence of the horseshoe will not be dealt with in this paper $[25,26]$; cf. references [14] and [25].

\subsection{CHAOTIC MOTIONS AND STRANGE ATTRACTING SETS}

In this final section we present a digital simulation for the parameter values $x_{0}=0$, $\alpha=0, r=0 \cdot 8$, and $\omega=2 \cdot 8$, for which an apparently persistent chaotic motion was observed. Similar motions appear to occur for large sets of parameter values in $\omega$ ranges for which the period $n$ single impact orbits are unstable. In Figure 17(a) we show a
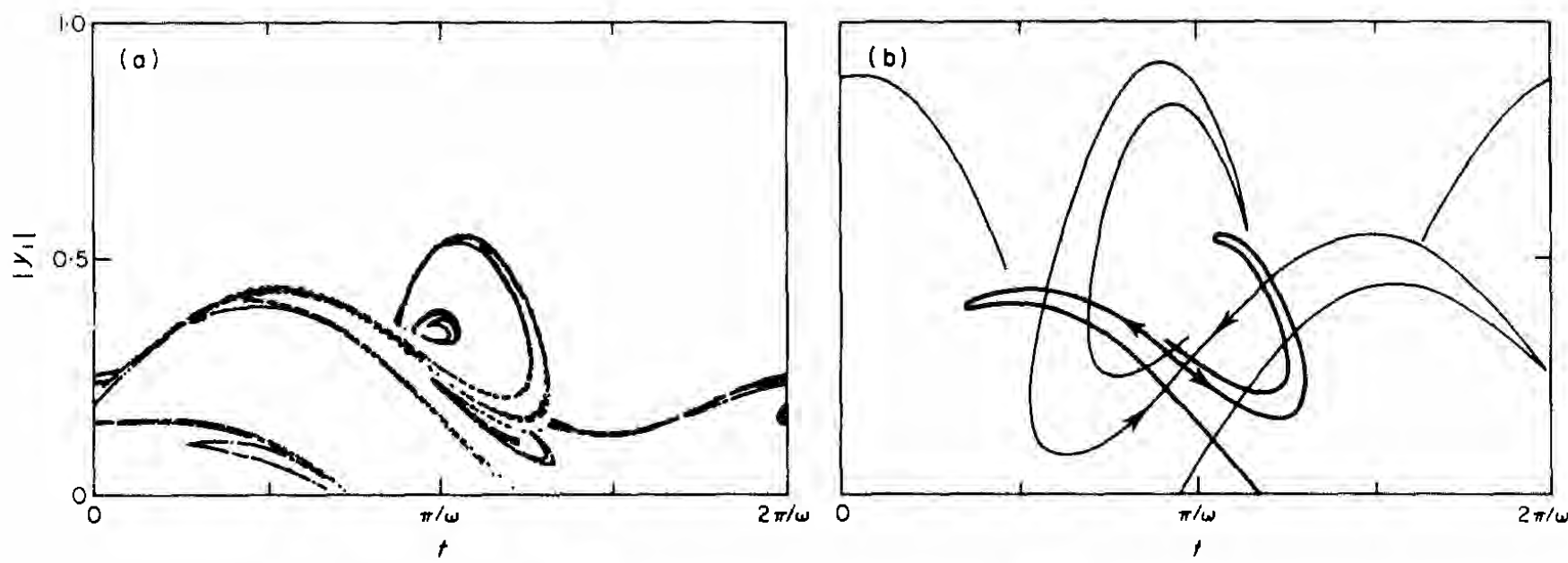

Figure 17. (a) Strange attracting set at $x_{0}=0 \cdot 0, \alpha=0, r=0.8$ and $\omega=2 \cdot 8$; (b) $W^{u}$ (heavy line) and $W^{s}$. (light line) at the same parameter values.

segment of a typical orbit of $\mathbf{P}$ containing 4000 points. Note that the orbit appears to lie on a well defined (set of ?) curves. Plotting the stable and unstable manifold of the saddle point (the $n=1$ orbit) in Figure 17(b) we see that this set of curves is indistinguishable from the unstable manifold. Similar observations have been made in many earlier papers (see references $[14,20,25]$, for example). The conjecture that there is an attracting set equal to the closure of this unstable manifold is irresistible, but we have not been able to prove it in this case (it can be proved for the Poincaré map of the Duffing equation [27]). However, even if it is true, Newhouse's work [28] shows that the attracting set may contain large, even infinite, sets of stable periodic orbits, so that it is not truly 
chaotic, for in such a situation almost all orbits will be asymptotically periodic, albeit with very long periods.

It is easy to establish the existence of an attracting set for the map P. We take the region $R=\{t, y \mid 0 \leqslant y \leqslant L\}$ in the cylindrical $(2 \pi / \omega$ periodic in $t)$ phase space. If $L$ is taken sufficiently large, then we can show that $\mathbf{P}(R) \subset R$, since a solution striking the wall $x=0$ with velocity $y_{0}>0$ at time $t_{0}$ immediately thereafter leaves with velocity $-r y_{0}$ and next strikes the wall at the velocity

$$
y_{2}=\frac{1}{1-\omega^{2}} \cos \omega t_{0} \sin \left(t_{2}-t_{0}\right)+\left(\frac{\omega}{1-\omega^{2}} \sin \omega t_{0}-r y_{0}\right) \cos \left(t_{2}-t_{0}\right)-\frac{\omega}{1-\omega^{2}} \sin \omega t_{2},
$$

obtained from equation (5), with $\alpha=0, \beta=1$ and $t_{1}=t_{0}$. Here $t_{2}-t_{0}$ is the (unknown) time of flight. From equation (71),

$$
y_{2} \leqslant \frac{1+2 \omega}{\left|1-\omega^{2}\right|}+r y_{0}
$$

and thus, provided $r<1$, one has

$$
y_{2}<y_{0}
$$

if $y_{0}$ is sufficiently large. We define the (closed) attracting set $\Omega$ as the intersection of all forward images of $R$ :

$$
\Omega=\bigcap_{n=0}^{\infty} \mathbf{P}^{n}(R)
$$

(Note that points on the lower boundary $y=0$ of $R$ may not be mapped into the interior, so $\Omega$ might contain points on this boundary.)

Now $\Omega$ might simply be a fixed point or a periodic orbit, but as shown in the previous section, it can also contain horseshoes. In this situation we refer to it as a strange attracting set. The orbit shown in Figure 17(a) is asymptotic to such a set, but as remarked above there is no guarantee that such an orbit might not be eventually periodic: the attracting set might contain stable high period orbits (it certainly does, for some parameter values, if the period doubling sequences accumulate as described).

In the case of one dimensional maps there is a fairly complete general theory and it has been shown that genuinely chaotic attracting sets, containing no stable periodic orbits, exist for large sets of such maps (cf. reference [17]). Unfortunately, very few results are available in the two dimensional case, and in general we have only digital evidence for the existence of strange attractors. The present example provides a little more evidence of this type.

We end with the remark that, if we let energy losses in the impact become large, then the coefficient of restitution $r$ tends to zero and the map becomes one dimensional (since the velocity $y$ returns to zero after every impact). Such a map is more amenable to analysis than the full two dimensional map, and we plan to study it in a subsequent paper.

\section{CONCLUSIONS AND DISCUSSION OF RESULTS}

In this paper we have studied a periodically forced single degree of freedom non-linear oscillator. The non-linearity is piecewise linear and thus explicit solutions are known on each side of the point of discontinuity in slope, $x_{0}$. This allows one to consider the motion as iterates of a map P, each iterate corresponding to the state crossing $x_{0}$ with velocity 
$y \geqslant 0$. Although P cannot be written out explicitly, and in fact is not "onto", one can compute its derivative DP, almost everywhere, i.e., except at points of discontinuity.

In the case of finite stiffness ratio we have used DP, with a simple assumption (equation (40)) regarding the times of flight on each side of $x_{0}$, to show that stable, single impact, period one orbits can undergo supercritical flip bifurcations [21]. This results in the appearance of a stable, two impact, period two orbit. Digital simulations of the system verify the analysis and also demonstrate that other bifurcations occur. However, these do not seem amenable to direct analysis. Investigations using numerical techniques could be employed for further study of these bifurcations.

When the stiffness ratio becomes large, the system is referred to as an impact oscillator $[15,16]$. Using a simple impact rule, we have analytically found single impact orbits of period $n$ and investigated their stability. Again, these single impact orbits undergo supercritical flip bifurcations and stable, period two, double impact orbits appear. We then used a digital simulation to study further bifurcations of this system. Further period doublings were found to occur in an accumulating sequence $[17,18]$. In the dissipationless case the existence of a complicated "chaotic" invariant set, a horseshoe, was demonstrated for the second iterate of the map $\mathbf{P}^{2}$ [25]. At a set of parameter values near those for which the horseshoe appears, a strange attracting set was observed. Orbits asymptotic to such a set are either non-periodic or of extremely long period.

The results obtained for the impact oscillator show qualitative agreement with digital simulations done by Thompson [15, 16]. Also, recent experiments by Robinson [9], using a circuit to model equations of this type, show many similar features. Especially interesting are the transitions Robinson found which seem to correspond to the singularities of our mapping P. Preliminary experimental results of our own, using a cantilever beam with a non-linear boundary condition (cf. reference [12]), verify that a physical system with finite stiffness ratio does in fact exhibit period doubling as well as subharmonics of order three. Equation (1) provides a model for such behavior.

This singular nature of the mapping $P$ induced by solutions of equation (1) is of interest. Discontinuous maps arise in these piecewise linear systems as well as in other non-linear problems such as relaxation oscillations [1,2]. Little is known of what occurs globally in such maps and further analysis should be done in this area.

\section{REFERENCES}

1. N. MINORSKY 1962 Nonlinear Oscillations. Huntington, New York: Krieger Publishing.

2. A. A. ANDRONOV, A. A. VITT and S. E. KHAIKEN 1966 Theory of Oscillators. Reading, Massachusetts: Addison Wesley.

3. S. MAEZAWA and S. FURUKAWA 1973 Bulletin of the Japanese Society of Mechanical Engineering 16, 931-941. Superharmonic resonance in piecewise linear systems.

4. R. DRAGONI and A. REPACI 1979 Mechanics Research Communications 6, 283-238. Influence of viscous-coulomb damping on a system with steps.

5. S. MAEZAWA 1961 Bulletin of the Japanese Society of Mechanical Engineering 4, 201-229. Steady forced vibrations of unsymmetrical piecewise linear systems.

6. S. MAEZAWA, H. KumANO and Y. MiNAKUCHI 1980 Bulletin of the Japanese Society of Mechanical Engineering 23, 68-75. Forced vibrations in an unsymmetric linear system excited by general periodic forcing functions.

7. K. KLOTTER 1953 in Symposium on Nonlinear Circuit Analysis, Polytechnic Institute of Brooklyn, April 1953, 234-257. Steady state vibrations in systems having arbitrary restoring and damping forces.

8. J. M. T. ThOmpson, A. R. BoKaIAN and R. Ghaffari 1982 Preprint, Department of Civil Engineering, University College, London. Subharmonic resonance of a bilinear oscillator with applications to moored marine systems. 
9. F. N. H. RoBINSON 1982 Preprint, Clarendon Laboratory, Oxford, U.K. An experimental study of the catastrophic behavior of a nonlinear differential equation.

10. T. WATANABE 1978 Journal of Mechanical Design 100, 487-491. Forced vibrations of continuous system with nonlinear boundary condition.

11. H. G. DAVIES 1980 Journal of Sound and Vibration 68, 479-487. Random vibration of a beam impacting stops.

12. F. C. MOON and S. W. SHAW 1983 (to appear) International Journal of Nonlinear Mechanics. Chaotic vibrations of a beam with nonlinear boundary condition.

13. M. SENATOR 1970 Journal of the Acoustical Society of America 47, 1390-1397. Existence and stability of periodic motions of a harmonically forced impacting system.

14. P. HOLMES 1982 Journal of Sound and Vibration 84, 173-189. The dynamics of repeated impact with a sinusoidally vibrating table.

15. J. M. T. THOMPSON 1982 Preprint, Department of Civil Engineering, University College, London. A strange attractor in the resonance of an impact oscillator.

16. J. M. T. THOMPSON and R. GHAFFARI 1982 Physics Letters 91A, 5-8. Chaos after period doubling bifurcations in the resonance of an impact oscillator.

17. P. COLLET and J. P. ECKMANN 1980 Iterated Maps on the Interval as Dynamical Systems, Progress in Physics, No. 1. Boston, Massachusetts: Birkhauser.

18. M. FEIGENBAUM 1979 Journal of Statistical Physics 19, 25-52. Quantitative universality for a class of nonlinear transformations.

19. J. P. MERIAM 1975 Dynamics New York: John Wiley and Sons, Inc.

20. P. J. Holmes 1979 Philosophical Transactions of the Royal Society A292, 419-448. A nonlinear oscillator with a strange attractor.

21. J. GUCKENHEIMER 1980 in Dynamical Systems, CIME Lectures Bressanone, Italy, June 1978, Progress in Mathematics No. 8, 115-231. Boston, Massachusetts: Birkhauser.

22. C. S. Hsu 1977 Advances in Applied Mechanics 17, 245-301. On nonlinear parametric excitation problems.

23. D. R. J. CHILlingworTh 1976 Differential Topology with a View to Applications. London: Pitman Press.

24. G. DAhLQUiST and Å. BJORK 1974 Numerical Methods (translated by N. Anderson). London: Prentice-Hall.

25. J. GUCKENHEIMER and P. HOLMES 1983 Nonlinear Oscillations, Dynamical Systems, and Bifurcations of Vector Fields. New York: Springer Verlag.

26. J. MOSER 1973 Stable and Random Motions in Dynamical Systems. Princeton University Press.

27. P. J. HOLMES and D. C. WHITLEY 1983 in Proceedings Year of Concentration in Partial Differential Equations and Dynamical Systems, University of Houston, Texas. On the attracting set of Duffing's equation; I: analytical methods for small force and damping.

28. S. E. NEWHOUSE 1980 in Dynamical Systems, CIME Lecture Bressanone, Italy, June 1978, Progress in Mathematics No. 8, 1-114. Boston, Massachusetts: Birkhauser.

29. J. CARR 1981 Applications of Centre Manifold Theory, Applied Mathematical Sciences No. 35. New York: Springer-Verlag.

\section{APPENDIX: NORMAL FORM AND CENTER MANIFOLD CALCULATIONS}

We outline here the calculations which determine the types of flip bifurcation (subor super-critical) which occur in the finite stiffness and the impact problems.

Defining local variables,

$$
\rho=\left(\begin{array}{c}
\xi \\
\eta
\end{array}\right)=\left(\begin{array}{c}
t-\bar{t} \\
y-\bar{y}
\end{array}\right)
$$

one can expand the mapping (20) near the period one point in a Taylor series,

$$
\rho=\left(\begin{array}{c}
\xi \\
\eta
\end{array}\right) \rightarrow\left(\begin{array}{ll}
f_{t} & f_{y} \\
g_{t} & g_{y}
\end{array}\right)\left(\begin{array}{c}
\xi \\
\eta
\end{array}\right)+\left(\begin{array}{c}
\frac{1}{2} f_{t t} \xi^{2}+f_{t y} \xi \eta+\frac{1}{2} f_{y y} \eta^{2}+\cdots \\
\frac{1}{2} g_{t t} \xi^{2}+g_{t y} \xi \eta+\frac{1}{2} g_{y y} \eta^{2}+\cdots
\end{array}\right),
$$


or

$$
\boldsymbol{\rho} \rightarrow \mathbf{D P p}+\left(\begin{array}{c}
R(\boldsymbol{p}) \\
S(\boldsymbol{p})
\end{array}\right)
$$

where the derivatives are evaluated at the periodic point. A similarity transformation

$$
\mathbf{z}=\left(\begin{array}{l}
u \\
v
\end{array}\right)=\mathbf{B}^{-1} \mathbf{p}
$$

puts equation (A2) into diagonalized (or normal) form,

$$
\mathrm{z} \rightarrow\left(\begin{array}{cc}
\lambda_{1} & 0 \\
0 & \lambda_{2}
\end{array}\right) \mathrm{z}+\left(\begin{array}{c}
P(\mathbf{z}) \\
Q(\mathbf{z})
\end{array}\right)
$$

where $\lambda_{1,2}$ are the eigenvalues of DP, $\mathbf{B}$ is the corresponding matrix of eigenvectors, and

$$
\left(\begin{array}{c}
P(\mathbf{z}) \\
Q(\mathbf{z})
\end{array}\right)=\mathbf{B}^{-1}\left(\begin{array}{c}
R(\mathbf{B z}) \\
S(\mathbf{B z})
\end{array}\right)
$$

At the bifurcation point $\lambda_{1}=-\bar{D}$ and $\lambda_{2}=-1$ (only flip bifurcations are considered here) so that

$$
\begin{gathered}
u \rightarrow-\bar{D} u+P(u, v)=-\bar{D} u+\frac{1}{2} P_{u u} u^{2}+P_{u v} u v+\frac{1}{2} P_{v v} v^{2}+\cdots, \\
v \rightarrow-v+Q(u, v)=-v+\frac{1}{2} Q_{u u} u^{2}+Q_{u v} v u+\frac{1}{2} Q_{v v} v^{2}+\cdots
\end{gathered}
$$

Since $|\bar{D}|<1$ there is linear contraction (locally) in the $u$ direction, but in the $v$ direction behavior depends on non-linear terms. A local center manifold [29] given by

$$
u=h(v)
$$

exists on which the local behaviour can be reduced, by substituting equation (A8) into equation (A7), to a one dimensional mapping, where $w$ is the co-ordinate on $h(v)$ :

$$
w \rightarrow-w+Q(h(w), w) .
$$

Equations (A6)-(A8) combine to give a functional equation for $h(v)$ :

$$
h(-v+Q(h(v), v))+\bar{D} h(v)-P(h(v), v)=0 .
$$

Expanding $h(v), P(u, v)$, and $Q(u, v)$ in Taylor series at the origin, one obtains, from equation (A10),

$$
h(v)=\left(\frac{\frac{1}{2} P_{v v}}{(1+\bar{D})}\right) v^{2}+O\left(|v|^{3}\right)
$$

Using equations (A9) and (A11) one then obtains

$$
w \rightarrow-w+\frac{1}{2} Q_{v v} w^{2}+\left[\frac{1}{6} Q_{v v v}+\frac{1}{2} Q_{u v} P_{v v} /(1+\bar{D})\right] w^{3}+O\left(|w|^{4}\right),
$$

which governs the local behavior of the two dimensional map at the bifurcation point. The second iterate of equation (A12) is

$$
w \rightarrow w-\left[\frac{1}{2} Q_{v v}^{2}+\frac{1}{3} Q_{v v v}+Q_{u v} P_{v v} /(1+\bar{D})\right] w^{3}+O\left(|w|^{4}\right),
$$

from which one sees that the stability of $w=0$ at the bifurcation point (which corresponds to the periodic point $(\bar{t}, \bar{y}))$ depends on the non-linear coefficient

$$
a=\frac{1}{2} Q_{v v}^{2}+\frac{1}{3} Q_{v v v}+Q_{u v} P_{v v} /(1+\bar{D})
$$


For $a<0 w=0$ is weakly unstable and one has a subcritical flip bifurcation. For $a>0 w=0$ is weakly stable and one has a supercritical flip bifurcation [21]. Note from equation (A14) that it is sufficient to show that

$$
\frac{1}{3} Q_{v v v}+Q_{u v} P_{v v} /(1+\bar{D})>0
$$

for supercriticality.

The coefficient $a$ depends on the terms $Q_{v v} Q_{u v}, P_{v v}$ and $Q_{v v v}$. The first three are linear combinations of the second derivatives of $f$ and $g$ and $Q_{v v v}$ is a linear combination of their third derivatives. The expressions for them are determined from equation (A5) and are quite lengthy.

In order that these bifurcations be non-degenerate (cf. reference [25]) the eigenvalue passing through -1 must do so with non-zero speed as the parameters are varied. If the bifurcation curves for the finite stiffness case (Figure 6) and the impact oscillator (Figure 11) are crossed transversally as the parameters change, then this non-degeneracy condition is met.

We now outline the normal form and center manifold calculations and show the results for these two cases.

\section{GENERAL SYSTEM (FINITE STIFFNESS)}

Here we consider the case $x_{0}=0, \alpha \ll 1, \omega=2 \omega_{n}=2 \Omega_{n}+O\left(\alpha^{2}\right)$ for simplicity. We also use the time of flight assumption given by equation (40). The second and third derivatives of $f$ and $g$ can be computed analytically and evaluated to $O\left(\alpha^{2}\right)$. They are complicated expressions and are not given here. The transformation (A5) is greatly simplified here by using equation (45) to obtain, from equations (28)-(31) (at the bifurcation point and on the period one orbit),

$$
\begin{gathered}
\partial f / \partial t_{0}=\partial t_{2} / \partial t_{0}=f_{t}=-\left(\Omega_{+} / \Omega_{-}\right) \mathrm{e}^{-2 \pi \alpha / \omega}+O\left(\alpha^{2}\right)=-1+O\left(\alpha^{2}\right), \\
\partial f / \partial y_{0}=\partial t_{2} / \partial y_{0}=f_{y}=O\left(\alpha^{2}\right), \\
\partial g / \partial t_{0}=\partial y_{2} / \partial t_{0}=g_{t}=\left[\left(\Omega_{-}^{2}-\Omega_{+}^{2}\right) / \Omega_{+} \Omega_{-}\right] \mathrm{e}^{-2 \pi \alpha / \omega} \bar{N}_{0}+O\left(\alpha^{2}\right), \\
\partial g / \partial y_{0}=\partial y_{2} / \partial y_{0}=g_{y}=-\left(\Omega_{-} / \Omega_{+}\right) \mathrm{e}^{-2 \pi \alpha / \omega}+O\left(\alpha^{2}\right)=-\bar{D}+O\left(\alpha^{2}\right)
\end{gathered}
$$

where $\bar{D}=\mathrm{e}^{-4 \pi \alpha / \omega}$. One sees that $t$ is an eigendirection (to be associated with $v$ ) and the similarity transformation $B$ is given by

$$
\mathbf{B}=\left[\begin{array}{cc}
0 & 1 \\
1-\bar{D} & (\bar{D}-1) / \mathrm{g}_{\mathrm{t}}
\end{array}\right],
$$

to order $\alpha^{2}$. The required terms in this case are

$$
\begin{gathered}
\frac{1}{2} Q_{v v}=\frac{1}{2} f_{t t}+n f_{t y}+\left(n^{2} / 2\right) f_{y y}, \quad Q_{u v}=m f_{t y}+m n f_{y y}, \\
\frac{1}{6} Q_{v v v}=\frac{1}{6} f_{t u t}+(n / 2) f_{t y}+\left(n^{2} / 2\right) f_{t y y}+\left(n^{3} / 6\right) f_{y y y}, \\
\frac{1}{2} P_{v v}=-(n / 2 m) f_{t t}-\left(n^{2} / m\right) f_{t y}-\left(n^{3} / 3 m\right) f_{y y}+(1 / 2 m) g_{t u}+(n / m) g_{t y}+\left(n^{2} / 2 m\right) g_{y y},
\end{gathered}
$$

where $m=1-\bar{D}$ and $n=g_{t} /(1+\bar{D})$ and all derivatives are evaluated on the periodic orbit. The coefficient $a$, given by equation (A14), was computed for a few values of $\tilde{\omega}$ for $\omega=2 \omega_{n}$ and $\alpha$ given by equation (46). It should be made clear that the calculation of a numerical value for $a$ requires knowledge of the fixed point value $(\bar{t}, \bar{y})$. This can be found only by use of the digital simulation. Thus the analytical work described above must be combined with results from the digital simulation in order to compute $a$. Table 
1 shows values of $a$ to $O\left(\alpha^{2}\right)$ for some sample values of $\tilde{\omega}$. In all cases $a>0$, and the bifurcations are consequently supercritical flip bifurcations as shown in Figure 5(a). This analysis verifies only a few discrete points on the curves of Figure 6 and in no way guarantees that all of the flip bifurcations in this problem are supercritical. However, no examples of subcritical bifurcations were observed in the digital simulations.

\section{IMPACT OSCILLATOR}

For the impact oscillator we restrict our attention to the case $x_{0}=0, \alpha=0$. Here the coefficient $a$ can be computed with no approximation and without use of the digital simulation. This is so because (1) one can find the bifurcation point exactly by using equation (66), (2) the fixed point is known exactly from equations (63) and (64), and (3) the times of flight are known exactly. The required terms here involve all of the second and third derivatives of $f$ and $g$; no simplifications occur as in the finite stiffness case, The matrix $B$ is

$$
\mathbf{B}=\frac{1}{r^{2}-1}\left[\begin{array}{cc}
-1-f_{t} & -f_{y} \\
f_{t}-r^{2} & f_{y}
\end{array}\right]
$$

and the required terms are

$$
\begin{gathered}
\frac{1}{2} Q_{v v}=k\left[-(p / 2) f_{t t}-p q f_{y t}-\left(p q^{2} / 2\right) f_{y y}+\frac{1}{2} g_{t t}+q g_{y t}+\left(q^{2} / 2\right) g_{y y}\right] \\
Q_{u v}=k\left[-p f_{t t}-p(p+q) f_{y t}-p^{2} q f_{y y}+g_{t t}+(p+q) g_{y t}+p q g_{y y}\right], \\
\frac{1}{6} Q_{v v v}=k\left[-(p / 6) f_{t t t}-(p q / 2) f_{t t y}-\left(p q^{2} / 2\right) f_{t y y}-\left(p q^{3} / 6\right) f_{y y y}\right. \\
\left.+\frac{1}{6} g_{t t t}+(q / 2) g_{t t y}+\left(q^{2} / 2\right) g_{t y y}+\left(q^{3} / 6\right) g_{y y y}\right] \\
\frac{1}{2} P_{v v}=k\left[(q / 2) f_{t t}+q^{2} f_{t y}+\left(q^{3} / 2\right) f_{y y}-\frac{1}{2} g_{t t}-q g_{t y}-\left(q^{2} / 2\right) g_{y y}\right]
\end{gathered}
$$

where $k=1 / f_{y}\left(r^{2}-1\right), p=-\left(r^{2}-f_{t}\right) / f_{y}, q=\left(-1-f_{t}\right) / f_{y}$, and all derivatives are again evaluated on the periodic orbit. Sample calculations were performed and the results are shown in Figure A1. As in the finite stiffness case, the bifurcations are supercritical flips.

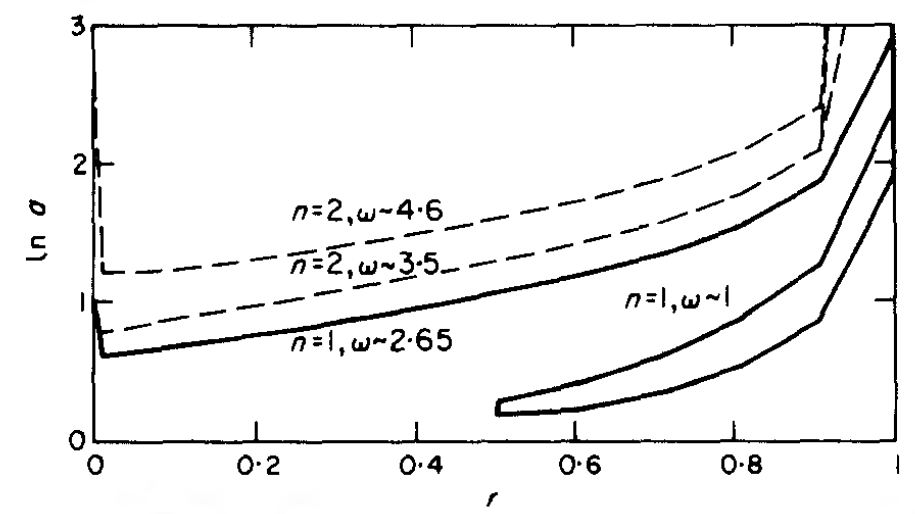

Figure A1. Plots of $\ln a$ vs. $r$, showing supercriticality for $n=1$ and 2 . 